\title{
Article
}

\section{The census of dense cores in the Serpens region from the Herschel Gould Belt Survey}

Fiorellino, E, Elia, D, André, Ph, Men'shchikov, A, Pezzuto, S, Schisano, E, Konyves, Vera, Arzoumanian, D, Benedettini, M, WardThompson, Derek, Bracco, A, Francesco, J Di, Bontemps, S, Kirk, Jason Matthew, Motte, F and Molinari, S

Available at http://clok.uclan.ac.uk/35532/

Fiorellino, E, Elia, D, André, Ph, Men'shchikov, A, Pezzuto, S, Schisano, E, Konyves, Vera, Arzoumanian, D, Benedettini, M et al (2021) The census of dense cores in the Serpens region from the Herschel Gould Belt Survey. Monthly Notices of the Royal Astronomical Society, 500 (4). pp. 4257-4276. ISSN 1365-2966

It is advisable to refer to the publisher's version if you intend to cite from the work. http://dx.doi.org/10.1093/mnras/staa3420

For more information about UCLan's research in this area go to http://www.uclan.ac.uk/researchgroups/ and search for <name of research Group>.

For information about Research generally at UCLan please go to http://www.uclan.ac.uk/research/

All outputs in CLoK are protected by Intellectual Property Rights law, including Copyright law. Copyright, IPR and Moral Rights for the works on this site are retained by the individual authors and/or other copyright owners. Terms and conditions for use of this material are defined in the policies page. 


\title{
The census of dense cores in the Serpens region from the Herschel Gould Belt Survey
}

\author{
E. Fiorellino ${ }^{\circledR}, 1,2,3,4 \star$ D. Elia ${ }^{\circledR}, 3$ Ph. André, ${ }^{5}$ A. Men’shchikov, ${ }^{5}$ S. Pezzuto ${ }^{\circledR}, 3$ E. Schisano ${ }^{\circledR}, 3$
} V. Könyves, ${ }^{6}$ D. Arzoumanian, ${ }^{7}$ M. Benedettini, ${ }^{3}$ D. Ward-Thompson, ${ }^{6}$ A. Bracco, ${ }^{8}$ J. Di Francesco,,${ }^{9,10}$ S. Bontemps, ${ }^{11,12}$ J. Kirk, ${ }^{6}$ F. Motte ${ }^{13}$ and S. Molinari ${ }^{\circledR 3}$

${ }^{1}$ Dipartimento di Fisica, Università di Roma 'Tor Vergata' Via della Ricerca Scientifica 1, I-00133 Roma, Italy

${ }^{2}$ INAF - Osservatorio Astronomico di Roma, via di Frascati 33, I-00078 Monte Porzio Catone, Italy

${ }^{3}$ INAF - Istituto di Astrofisica e Planetologia Spaziali (IAPS), via Fosso del Cavaliere 100, I-00133 Roma, Italy

${ }^{4}$ ESO/European Southern Observatory, Karl-Schwarzschild-Str. 2, D-85748 Garching bei Munchen, Germany

${ }^{5}$ Laboratoire d'Astrophysique (AIM), CEA/DRF, CNRS, Université Paris-Saclay, Université Paris Diderot, Sorbonne Paris Cité, F-91191 Gif-sur-Yvette,

France

${ }^{6}$ Jeremiah Horrocks Institute, University of Central Lancashire, Preston PR12HE, UK

${ }^{7}$ Instituto de Astrofísica e Ciências do Espaço, Universidade do Porto, CAUP, Rua das Estrelas, PT4150-762 Porto, Portugal

${ }^{8}$ Rudjer Bošković Institute, Bijenička cesta 54, 10000 Zagreb, Croatia

${ }^{9}$ Department of Physics and Astronomy, University of Victoria, PO Box 355, STN CSC, Victoria, BC, V8W 3P6, Canada

${ }^{10}$ National Research Council Canada, 5071 West Saanich Road, Victoria, BC, V9E 2E7, Canada

${ }^{11}$ Univ. Bordeaux, LAB, UMR5804, F-33270 Floirac, France

${ }^{12}$ CNRS, LAB, UMR5804, F-33270 Floirac, France

${ }^{13}$ University Grenoble Alpes, Centre National de la Recherche Scientifique (CNRS), Institut de Planétologie et d'Astrophysique de Grenoble, F-38000 Grenoble, France

Accepted 2020 October 28. Received 2020 October 27; in original form 2020 July 28

\begin{abstract}
The Herschel Gould Belt survey mapped the nearby $(d<500 \mathrm{pc})$ star-forming regions to understand better how the prestellar phase influences the star formation process. Here, we report a complete census of dense cores in a $\sim 15 \mathrm{deg}^{2}$ area of the Serpens star-forming region located between $d \sim 420$ and $484 \mathrm{pc}$. The PACS and SPIRE cameras imaged this cloud from 70 to $500 \mu \mathrm{m}$. With the multiwavelength source extraction algorithm getsources, we extract 833 sources, of which 709 are starless cores and 124 are candidate protostellar cores. We obtain temperatures and masses for all the sample, classifying the starless cores in 604 prestellar cores and 105 unbound cores. Our census of sources is 80 per cent complete for $M>0.8 \mathrm{M}_{\odot}$ overall. We produce the core mass function (CMF) and compare it with the initial mass function (IMF). The prestellar CMF is consistent with lognormal trend up to $\sim 2 \mathrm{M}_{\odot}$, after which it follows a power law with slope of $-2.05 \pm 0.34$. The tail of its CMF is steeper but still compatible with the IMF for the region we studied in this work. We also extract the filaments network of the Serpens region, finding that 81 per cent of prestellar cores lie on filamentary structures. The spatial association between cores and filamentary structure supports the paradigm, suggested by other Herschel observations, that prestellar cores mostly form on filaments. Serpens is confirmed to be a young, low-mass and active star-forming region.
\end{abstract}

Key words: stars: formation - ISM: clouds - ISM: individual (Serpens) - ISM: structure-infrared: ISM - submillimetre: ISM.

\section{INTRODUCTION}

Solar-type stars form from the collapse of compact structures called prestellar cores, but how the physical properties of stars are determined during the prestellar core phase is still a matter of debate. We want to contribute to this debate by analysing the Herschel Gould Belt Survey (HGBS; André et al. 2010) observations of the Serpens star-forming region, which is deeply described in some detail in Section 1.1.
The HGBS is one of the largest projects with the Herschel Space Observatory (Pilbratt et al. 2010), whose main objectives are to take a deep census of prestellar cores in nearby clouds, determine a reliable prestellar CMF, and investigate the link between the CMF and the IMF in detail, based on the observations of the nearest ( $d \leq 500 \mathrm{pc})$ star-forming clouds in the Milky Way.

Herschel observed with its far-infrared and sub-millimetre cameras PACS (Poglitsch et al. 2010) and SPIRE (Griffin et al. 2010), respectively, with unprecedented sensitivity and resolution in the range between 70 and $500 \mu \mathrm{m}$. In this wavelength range the spectral energy distribution (SED) of cold dust $(T \sim 10 \mathrm{~K}$ ) is expected to have its peak $(\lambda \sim 250 \mu \mathrm{m})$. The Herschel sensitivity makes it possible to observe sources never detected before in the Serpens Main region, 
allowing a more complete and accurate sampling of the Serpens Main core population compared to the previous studies based on statistics of few tens of objects. Moreover, the Herschel multiwavelength analysis enables to obtain both temperature and mass of each core from its SED, producing more accurate physical values with respect to single-wavelength pre-Herschel works that necessarily assumed a common temperature for the entire sample of cores (Testi \& Sargent 1998; Enoch et al. 2007).

The twofold purpose of our work, as a part of the HGBS project, is to characterize the earliest stages of star formation in the Serpens region and to verify whether accompanying results about its prestellar core mass function (CMF) are in agreement or not with ones obtained for other clouds and with previous studies of the Serpens Main subregion.

The paper is organized as follows: in the rest of this section, we introduce the Serpens area surveyed by Herschel (Section 1.1) and the concept of the CMF (Section 1.2), respectively. In Section 2, we describe the observations and data reduction. In Section 3, we present temperature and column density maps. In Section 4 , we set out the detection of the sources, the production of the catalogue, the fit of a modified blackbody to SEDs, and the analysis of the obtained core physical parameters. In Section 5, we report and discuss the CMF of prestellar cores. In Section 6, we focus on the analysis of the Serpens Main sub-region. In Section 7, we illustrate the filamentary structure of this region and its relationship with the prestellar core spatial distribution. Finally, in Section 8 we draw conclusions from this work.

\subsection{The Serpens region}

The Serpens region extends over several square degrees around the variable star VV-Ser. It is part of the Aquila Rift cloud complex, through the middle of the plane of the Milky Way. It was firstly recognized as a star formation site by Strom, Grasdalen \& Strom (1974).

Fig. 1 shows the region mapped by Herschel, a wider region (the blue box region) with respect to that ascribed to the Serpens in the literature (i.e. Serpens Main, the 'Green box' region in Fig. 1). We will refer to this sub-region as Serpens Main in the following. Therefore in this section, when reviewing previous literature about Serpens, we write essentially about Serpens Main, while the Herschel observations presented in this paper also include a large portion the easternmost part of the Aquila Rift. We referred to this last portion of the sky as Aquila East, which has been poorly studied so far. A portion of the Aquila East region has been analysed by Herczeg et al. (2019) in their overview of the structure of Serpens/Aquila East complex with Gaia data. This region was also observed as a part of the Spitzer Gould Belt survey, whose catalogue of young stellar objects (YSOs) was presented by Dunham et al. (2015). Finally, Aquila East was surveyed in CO by Nakamura et al. (2017). This work represents an occasion to give a further look to early stages of star formation in this region, thanks to Herschel far-infrared/sub-mm observations.

Notice that the portion of the Aquila Rift analysed in Könyves et al. (2015) is distinct from the field studied in this work. In the following, we will name this region Aquila Main to avoid confusion between these two different parts of the area.

The mass of Serpens Main region has been estimated by several authors, but based on different distance assumptions (see Section 1.1.1), different tracers and corresponding calculation techniques, and, finally, considering different areas. The comparison between these mass values and our results is reported in Section 3.
The Serpens Main region contains two sub-clumps (Testi et al. 2000; Olmi \& Testi 2002): the north-west (NW) one, called Serpens Core or Cluster A, and the south-east (SE) one, called Serpens G366, or Cluster B (Djupvik et al. 2006; Harvey et al. 2006; Levshakov et al. 2013). Cluster B has more prominent filaments and more complicated kinematics than Cluster A. Moreover, it has a higher degree of hierarchy (Lee et al. 2014). Between these two clusters, Enoch et al. (2007) found an elongated filament that contains several bright sources.

The Serpens complex is generally classified in the literature as a low-mass star forming region (Enoch et al. 2007; Eiroa, Djupvik \& Casali 2008). Recently, Roccatagliata et al. (2015) suggested that Serpens Main could host high-mass star formation. However, Nakamura et al. (2017), considering column density thresholds (Krumholz \& McKee 2008; Kauffmann \& Pillai 2010, see Section 4.1), conclude that none of the clumps presents necessary conditions for the formation of high-mass stars. According to Casali, Eiroa \& Duncan (1993) and Kaas et al. (2004), star formation in the Serpens Main region occurred in several steps, because of the presence of evolved stars, young stellar objects, prestellar cores and a relevant amount of dust which in turn traces the presence of molecular gas available for future star formation activity (Nakamura et al. 2017). Nevertheless, Testi et al. (2000) described a different scenario in which there is no need for more than one star formation event.

The filamentary structure of Serpens Main is composed of both gravitationally supercritical and subcritical filaments (Lee et al. 2014). Mapping Serpens Main in $3 \mathrm{~mm}$ lines, these authors found six filaments in the SE subcluster, whose lengths range from 0.17 to $0.33 \mathrm{pc}$, and widths from 0.03 to $0.05 \mathrm{pc}$, respectively. In addition, several YSOs have formed along two filaments with supercritical masses-per-unit-length, while filaments with nearly critical mass-per-unit-length are not associated with YSOs, suggesting that stars formed preferentially in gravitationally unstable filaments. Roccatagliata et al. (2015) identified nine filaments in Herschel maps, affirming that their simulations could explain the filament morphology but not the kinematics. For example, while the simulated filaments of Serpens Main are likely turbulence-dominated regions, for which collapse into smaller structures is not expected, the observed filaments are self-gravitating structures that will fragment into cores.

\subsubsection{The adopted distance}

As described in detail by Eiroa et al. (2008), the determination of the distance of the Serpens region is tricky, indeed distances between 200 and 700 pc are found in the literature (Chavarria-K. et al. 1988; Zhang et al. 1988; Dzib et al. 2010, 2011).

First of all, it makes sense to search for a global distance of the region (with possible secondary differences among sub-regions), as suggested originally by line emission surveys: channel maps presented in Prato, Rice \& Dame (2008) indeed indicate that COemission is kinematically connected throughout the region.

Recently, Ortiz-León et al. (2017, 2018), based on VLBI astrometry of a number of young stellar objects (YSOs) in the Serpens/Aquila East complex, concluded that Serpens Main and Aquila Rift clouds, and in particular Aquila East, are physically associated and lie at a common distance of $d=436.0 \pm 9.2 \mathrm{pc}$.

More recently, Zucker et al. (2019) reported the distances of several nearby molecular clouds (including the region we study here), by combining line-of-sight extinctions from new near-infrared (NIR) star photometry with Planck continuum maps at $353 \mathrm{GHz}$ (Planck 


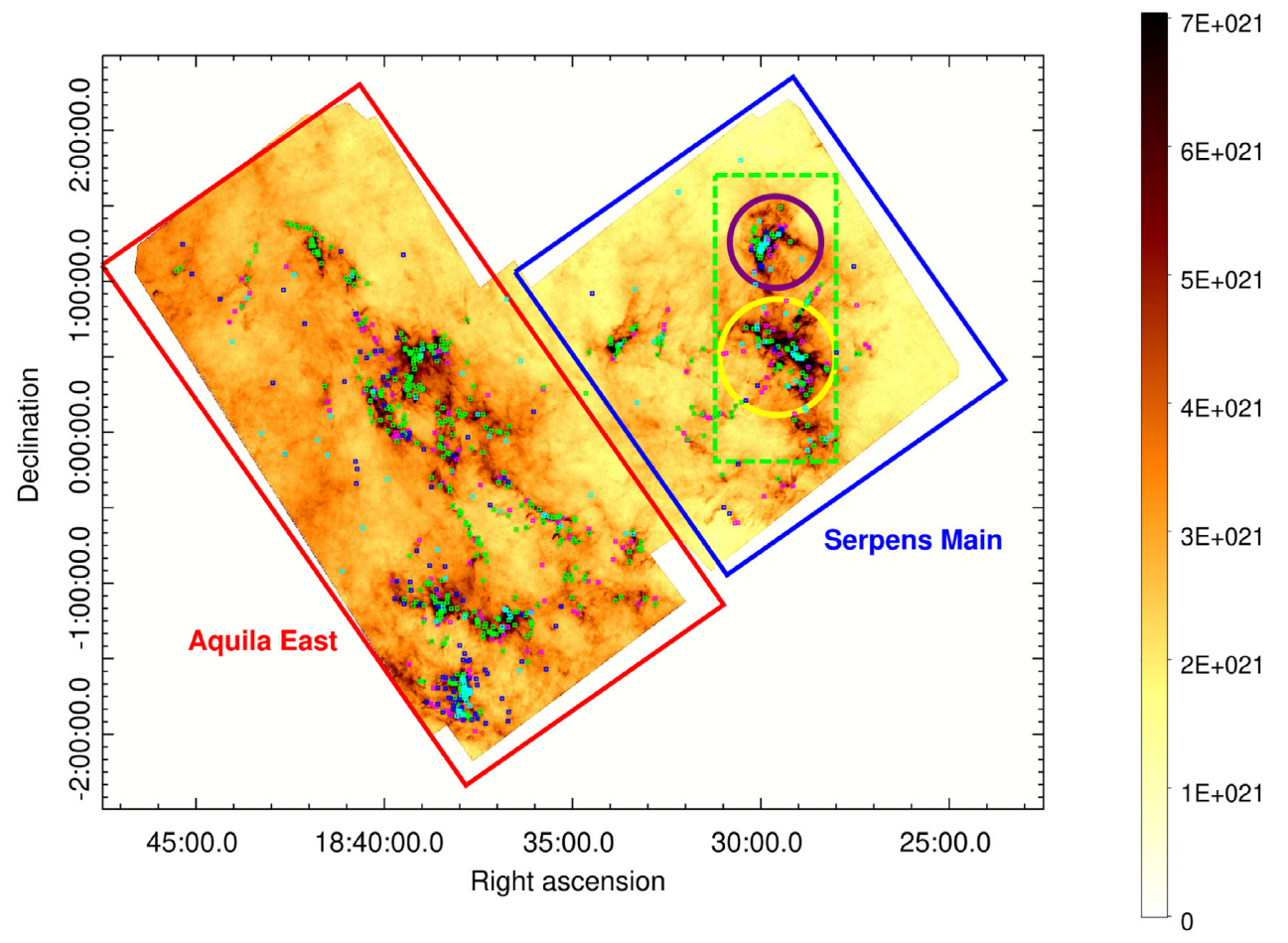

Figure 1. High-resolution (18.2 arcsec) column density map of the Serpens complex observed by Herschel (Section 3), with the sources extracted by getsources algorithm (see Section 2). Protostellar cores, unbound starless cores, robust, and candidate prestellar cores are plotted with cyan, blue, green, and magenta symbols, respectively. The colour bar reports the column density in $\mathrm{cm}^{-2}$. The blue square encompasses the Serpens Main in which Cluster A (purple circle) and Cluster B (yellow circle) are located (Djupvik et al. 2006; Harvey et al. 2006; Levshakov et al. 2013); the red rectangle contains the Aquila East. The green dashed box shows the part of the Serpens region we select to compare our results about Serpens Main with those from previous studies (Section 6).

Collaboration XI 2014) and Gaia parallaxes. They conclude that the Serpens/Aquila Rift clouds form a single coherent complex, at a similar distance, with single regions having possible peculiar distances, resulting in a dispersion along the line of sight of about $50 \mathrm{pc}$. Having to quote a global distance for such a complex, they determine $d=484 \pm 28$ pc. However, for the Serpens Main area only, Zucker et al. (2019) estimate a specific distance of $420 \mathrm{pc}$ (with 5-6 per cent uncertainty) which is in agreement with Ortiz-León et al. (2017) result.

A quick check we made with the Gaia data release 2 (Gaia-dr $2^{1}$ ) supports this distance estimate for Serpens Main: i.e. the VV-Ser parallax measured is $p=2.38 \pm 0.046$ marcsec, which corresponds to a distance of about $d=1 / p \simeq 420.0 \pm 8.1 \mathrm{pc}$ (in turn consistent, within the errors, also with that of Ortiz-León et al. 2018).

Summarizing, the final decision for this paper consists in studying the whole surveyed area as a unique, coherent, physically connected star-forming complex, composed by two main sub-regions located at two single different distances: 484 pc for Aquila East, and $420 \mathrm{pc}$ for Serpens Main, respectively. The Serpens Main sub-region is further discussed separately in Section 6.

\subsection{The prestellar core mass function}

A typical observable useful to investigate the link between the properties of the core population and the characteristics of the star formation in a given region is the statistical mass distribution of cores (core mass function, $\mathrm{CMF}$ ), which can be compared with the initial

${ }^{1}$ https://gea.esac.esa.int/archive/ mass function (IMF) of stars (e.g. Motte, Andre \& Neri 1998; Testi \& Sargent 1998; Offner et al. 2014).

The IMF power-law behaviour was first computed by Salpeter (1955), who found

$\frac{\mathrm{d} N}{\mathrm{~d} M} \propto\left(\frac{M}{\mathrm{M}_{\odot}}\right)^{-\gamma}$

with $\gamma=2.35$ for $-0.4 \leq \log \left(\frac{M}{M_{\odot}}\right)<1.0$. Decades later, Motte et al. (1998) and Testi \& Sargent (1998) found a similarity in shape between the IMF and the CMF in the Ophiucus and Serpens Main star-forming regions, respectively. Indeed, the power-law slopes were consistent, but over a different mass range. In particular for Serpens Main, Testi \& Sargent (1998) found a slope $\gamma=2.1$, while subsequently Enoch et al. (2007) found $\gamma=2.6$. Only the first value is consistent with the observational IMF slope and with that predicted by turbulent fragmentation simulations $\gamma=2.33$ (e.g. Padoan $\&$ Nordlund 2002). The similarity between the power-law tail of the IMF and of CMF suggests that a direct link might exist between the star properties and the fragmentation process. Subsequent works improved the knowledge of the IMF, still observing the relationship with the CMF, not only for the power-law tail trend at high masses, but also for the lognormal behaviour of both mass functions at low masses (Kroupa 2001; Chabrier 2005; Lee et al. 2014). Recent papers based on the HGBS seem to confirm the similarities between CMF and IMF for young star-forming regions (André et al. 2010; Polychroni et al. 2013; Könyves et al. 2015; Marsh et al. 2016). One of the purposes of this paper is precisely to verify whether new Herschel observations are consistent with previous results, both for the specific Serpens region and for general comparison between CMF and IMF (see Sections 5 and 6). 


\section{OBSERVATIONS}

Herschel observations of the Serpens/Aquila East complex include two sub-regions: a box of approximately $\sim 2.2 \times 2.5$ square degrees to the east, centred on RA $=18^{\mathrm{h}} 29^{\mathrm{m}} 32^{\mathrm{s}}$, Dec. $=+0^{\circ} 40^{\prime} 42^{\prime \prime}$ that includes Serpens Main; the box of approximately $\sim 2.3 \times 4.4 \mathrm{deg}^{2}$, centred on RA $=18^{\mathrm{h}} 38^{\mathrm{m}} 48^{\mathrm{s}}$, Dec. $=-0^{\circ} 00^{\prime} 37^{\prime \prime}$, is the Aquila East (see Fig. 1). Observations were taken on 2010 October 16-18, ObsIDs 1342206676/95 and 1342206694/96 for Serpens Main and Aquila East, respectively. Data were taken using PACS at $70 \mu \mathrm{m}$ and $160 \mu \mathrm{m}$, and SPIRE at 250, 350, and $500 \mu \mathrm{m}$. Two orthogonal scan maps were performed in parallel mode at $60 \operatorname{arcsec~s}^{-1}$. Data taken during the turnarounds were included.

The data reduction procedure was different for PACS and SPIRE observations. SPIRE data were reduced entirely with HIPE Version 10.1, provided by Herschel Science Center, producing the images using its 'naive' map-making procedure and destriper module (Herschel Science Ground Segment Consortium 2011). Differently, PACS data were reduced with HIPE v10.1 and images were obtained using UNIMAP Version 6.4.1 (Piazzo et al. 2015) for the map making and destriping phase. The pixel size of the maps has been set to 3 arcsec at $70 \mu \mathrm{m}, 4 \operatorname{arcsec}$ at $160 \mu \mathrm{m}, 6 \operatorname{arcsec}$ at $250 \mu \mathrm{m}, 10 \operatorname{arcsec}$ at $350 \mu \mathrm{m}$, and $14 \operatorname{arcsec}$ at $500 \mu \mathrm{m}$, respectively. The FWHM beam sizes are $8.4,13.5,18.2,24.9$, and 36.3 arcsec for the maps at 70 , $160,250,350$, and $500 \mu \mathrm{m}$, respectively. ${ }^{2}$

The Herschel maps have an arbitrary zero-level intensity, so the intensity in each pixel is known except for a constant term. This offset was evaluated assuming a dust model exploiting Planck and IRAS data, using a method similar to the one described by Bernard et al. (2010) to obtain the Planck median $\langle P>$, and subtracting the Herschel median $\langle H\rangle$. The estimated offsets are reported in Table 1. To analyse the entire region, we composed the calibrated maps of Serpens Main and Aquila East into mosaic maps right after the map making by merging the two observations in a single map.

\section{PHYSICAL ANALYSIS OF THE SERPENS REGION}

\subsection{Column density \& temperature maps}

To compute temperature and $\mathrm{H}_{2}$ column density maps, we assumed that the interstellar dust emits like a modified blackbody in the farIR:

$F_{v}=\frac{\kappa_{v} M}{d^{2}} B_{v}(T)$

(e.g. Elia \& Pezzuto 2016), where $F_{\nu}$ is the flux emitted by the dust at the frequency $v, \kappa_{v}$ is the dust opacity, $M$ is the mass of emitting material for a given pixel, $B_{v}(T)$ is the Planck function at temperature $T$, and $d$ is the source distance.

The dust opacity is usually parametrized as

$\kappa_{v}=\kappa_{0}\left(\frac{v}{v_{0}}\right)^{\beta}$

in the far-IR, where $\kappa_{0}$ is a reference value estimated at $\nu_{0}$, and $\beta$ is the dust opacity index. In this work, we use $\kappa_{0}=0.1 \mathrm{~cm}^{2} \mathrm{~g}^{-1}$, which already accounts for the gas-to-dust ratio of 100, at $\lambda_{0}=c / \nu_{0}=$

\footnotetext{
${ }^{2} \mathrm{http} / / /$ gouldbelt-herschel.cea.fr/archives. The page at this link contains all the Serpens/Aquila East products, among other HGBS regions: the FITS maps of the region at individual PACS/SPIRE wavelengths $(70-500 \mu \mathrm{m})$, as well as column density and temperature FITS images.
}

Table 1. Planck and Herschel emission medians (for both Serpens Main and Aquila East), and final offsets obtained by the difference between them.

\begin{tabular}{lcccc}
\hline $\begin{array}{l}\text { Band } \\
\mu \mathrm{m}\end{array}$ & Region & $\begin{array}{c}<P> \\
\left(\mathrm{MJy} \mathrm{sr}^{-1}\right)\end{array}$ & $\begin{array}{c}<H> \\
\left(\mathrm{MJy} \mathrm{sr}^{-1}\right)\end{array}$ & $\begin{array}{c}\text { Offset } \\
\left(\mathrm{MJy} \mathrm{sr}^{-1}\right)\end{array}$ \\
\hline 70 & $\mathrm{SM}$ & 25.9 & 14.1 & 11.9 \\
& $\mathrm{AE}$ & 54.0 & 12.6 & 41.4 \\
160 & $\mathrm{SM}$ & 196.0 & 57.0 & 139.0 \\
& $\mathrm{AE}$ & 314.0 & 55.2 & 258.8 \\
250 & $\mathrm{SM}$ & 126.4 & 1.1 & 125.3 \\
& $\mathrm{AE}$ & 194.8 & 3.6 & 191.2 \\
350 & $\mathrm{SM}$ & 62.2 & 0.6 & 62.7 \\
& $\mathrm{AE}$ & 95.2 & 1.8 & 93.4 \\
500 & $\mathrm{SM}$ & 26.9 & 0.6 & 26.3 \\
& $\mathrm{AE}$ & 26.9 & 0.6 & 38.9 \\
\hline
\end{tabular}

$300 \mu \mathrm{m}$ (Hildebrand 1983) and $\beta=2$, for uniformity with other HGBS works (Kirk et al. 2013; Könyves et al. 2015; Benedettini et al. 2015), based on Roy et al. (2014), in which it is shown that this assumption is good within better than $\sim 50$ per cent in the bulk of molecular clouds.

The choice of $\kappa_{0}$ and $\beta$ can be somehow critical (see e.g. Elia et al. 2017; Alton et al. 2004, for a discussion on $\kappa_{0}$ and $\beta$, respectively), since it has consequences on the column density or masses derived through equation (2). As $\kappa_{0}$ increases the mass linearly decreases, while at the large wavelengths, where $\left(\nu / \nu_{0}\right)<1$, if $\beta>2$, then the mass increases with respect the estimate we give in Section 3.2.

The temperature and column density maps were obtained pixel by pixel by fitting a modified blackbody (equation 2) to fluxes at $160,250,350$, and $500 \mu \mathrm{m}$, after re-projecting each map over the $500 \mu \mathrm{m}$ grid. The $70 \mu \mathrm{m}$ map was not used to produce the column density map because the parametrization in equation (2) is valid only for $\lambda \gg 100 \mu \mathrm{m}$, while at shorter wavelengths the dust is not optically thin, and both cold and hot dust components are present. Note that the same technique is also applied to the SEDs of cores to derive core masses and temperatures (Section 4.1), which however, differently from this case, are built with background-subtracted and integrated flux densities. This difference can lead to differences between quantities derived in this way for cores, and those of the pixels corresponding to core locations (see e.g. Elia et al. 2013; Kirk et al. 2013; Könyves et al. 2015). We refer the reader to Section 4.1 for further details.

The temperature map is shown in Fig. 2. The estimated temperature ranges from 11.5 and $21.4 \mathrm{~K}$, with remarkable differences between the eastern (Aquila East region) and western (Serpens Main region) side of the complex. This gradient is probably due to a more efficient radiation shielding by the denser and colder $(T<16 \mathrm{~K})$ clusters in Serpens Main, which is probably a consequence of the fact that the Serpens Main is farther than the Aquila East from the Galactic Plane. We present the temperature distribution in Fig. 3, splitting it between the two sub-regions. The Aquila Rift is warmer and includes all the regions for which we estimated $T>19 \mathrm{~K}$. Its average temperature is $17.5 \mathrm{~K}$, dropping to $\sim 16 \mathrm{~K}$ only in the regions at higher density. We find that, as expected, temperatures are comparable with those derived by Könyves et al. (2015) in the Aquila Main region, ranging from 14 to $22 \mathrm{~K}$.

We assumed that the gas composition was primordial and we compute the column density values using

$N_{\mathrm{H}_{2}}=\frac{M}{\mu_{\mathrm{H}_{2}} m_{\mathrm{H}}} \frac{1}{A_{\text {pix }}}$, 


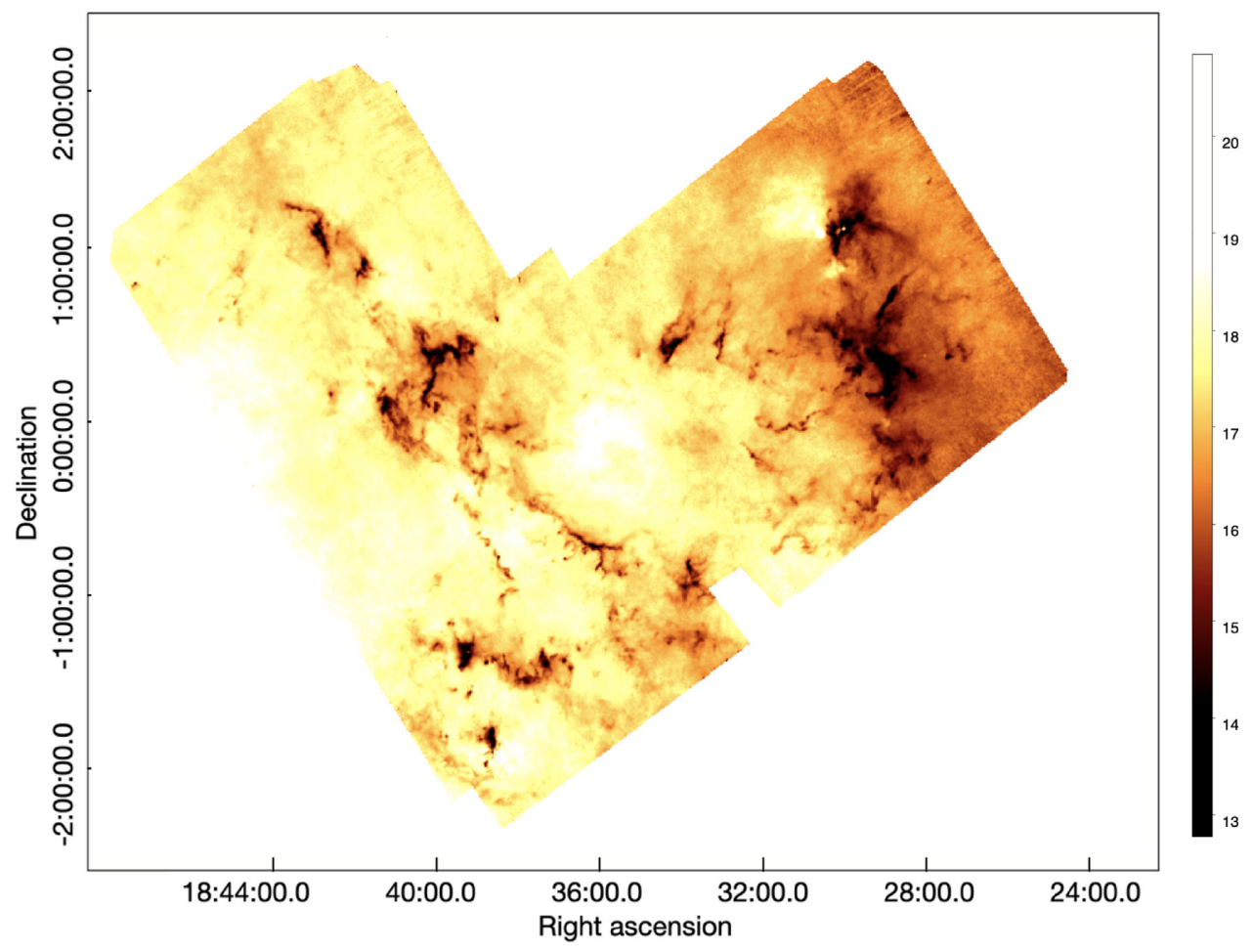

Figure 2. Temperature map of the Serpens region. The colour bar reports the temperature in K.

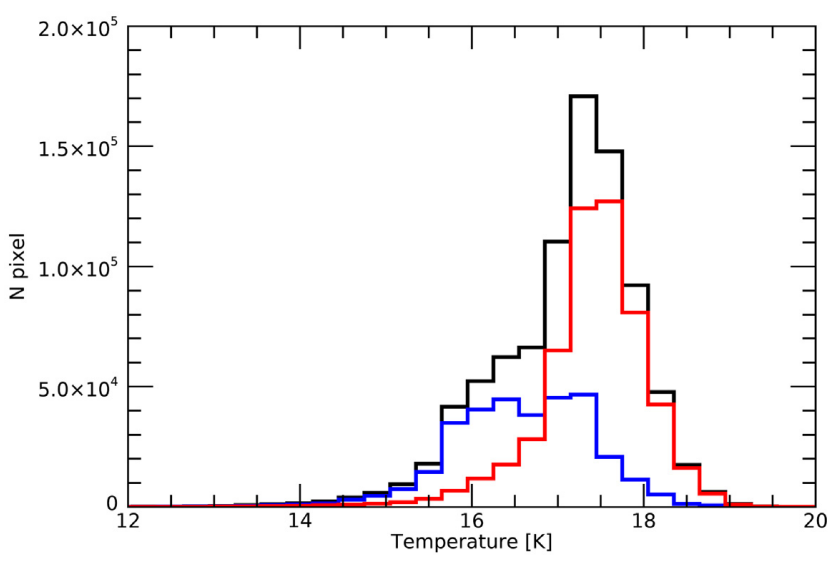

Figure 3. Temperature distributions of the Herschel Serpens region (black), see Fig. 2. The total distribution shows a swelling at about $16-17 \mathrm{~K}$ and a peak at about 17-18 K, corresponding to the peaks of the sub-regions: the highest at $\sim 17.5 \mathrm{~K}$, which is due to the Aquila East (red) contribution; the lowest at $\sim 16.5 \mathrm{~K}$, mostly due to the Serpens Main contribution (blue).

where $\mu_{\mathrm{H}_{2}}=2.8$ is the mean molecular weight (Kauffmann et al. 2008), $m_{\mathrm{H}}$ is the hydrogen mass, and $A_{\text {pix }}$ is the pixel area of the $500 \mu \mathrm{m}$ map.

We computed the optical depths in the Serpens region from the column density map whose pixel size was degraded to 5 arcmin, namely the resolution of the Planck map at $\lambda=850 \mu \mathrm{m}$, using the equation:

$\tau_{v}=\kappa_{v} \mu m_{\mathrm{H}} N_{\mathrm{H}_{2}}$

and compared the results with the Planck direct $\tau$ observations. We found our values are in agreement within $1 \sigma$, with the mean $\tau_{\mathrm{H}} / \tau_{\mathrm{P}}=$ 0.81 , the median $\tau_{\mathrm{H}} / \tau_{\mathrm{P}}=0.98$ and the standard deviation $\sigma\left(\tau_{\mathrm{H}} / \tau_{\mathrm{P}}\right)=$
0.39 , where $\tau_{\mathrm{H}}$ and $\tau_{\mathrm{P}}$ are the optical depths obtained by Herschel and Planck observations, respectively. This agreement confirms the consistency of the zero-level offsets derived from Planck data and applied to the Herschel maps (see Section 2).

As in previous HGBS works, we also determined a 'highresolution' column density map, as shown in Fig. 1, based on a multiscale decomposition of the imaging data, as described in appendix A of Palmeirim et al. (2013), and a 'temperature-corrected' map, based on the colour-temperature map of 160 and $250 \mu \mathrm{m}$, built to reduce the effects of strong, anisotropic temperature gradients present in parts of the observed fields (Könyves et al. 2015). These two maps are included in the HGBS source extraction process through the getsources algorithm (see Section 4).

The distribution of pixel column densities for Serpens cloud is displayed in Fig. 4. We will refer to this as the probability density function (PDF), even though the pure pixel distribution is not strictly a PDF, since this term is typically used in the context of continuous random variables, while we build it from a discrete set of data. The low-density part of the PDF behaves as a lognormal function, which is usually interpreted as the presence of a turbulence-dominated environment (Kainulainen et al. 2011). Accordingly, we fit the low density part with a lognormal, finding that our data are well fitted for $A_{\mathrm{V}} \lesssim 3$. In particular, a departure of the PDF from lognormal behaviour is seen around two bins that correspond to $A_{\mathrm{V}} \sim 4$ and 7 , respectively. The presence of an excess at high column density bins with respect to a lognormal behaviour is generally explained with a gravity-dominated scenario (Federrath 2013; Schneider et al. 2013), with presence of overdensities such as filaments and cores. This is found also if the two sub-regions are considered separately, with Serpens Main showing a more extended high-density tail (up to $\sim 10^{23} \mathrm{~cm}^{-2}$ ) compared to Aquila East (up to $\sim 5 \times 10^{22} \mathrm{~cm}^{-2}$ ).

Finally, we also notice that the Serpens PDF is similar to the Aquila Main PDF (Schneider et al. 2013; Könyves et al. 2015), since 


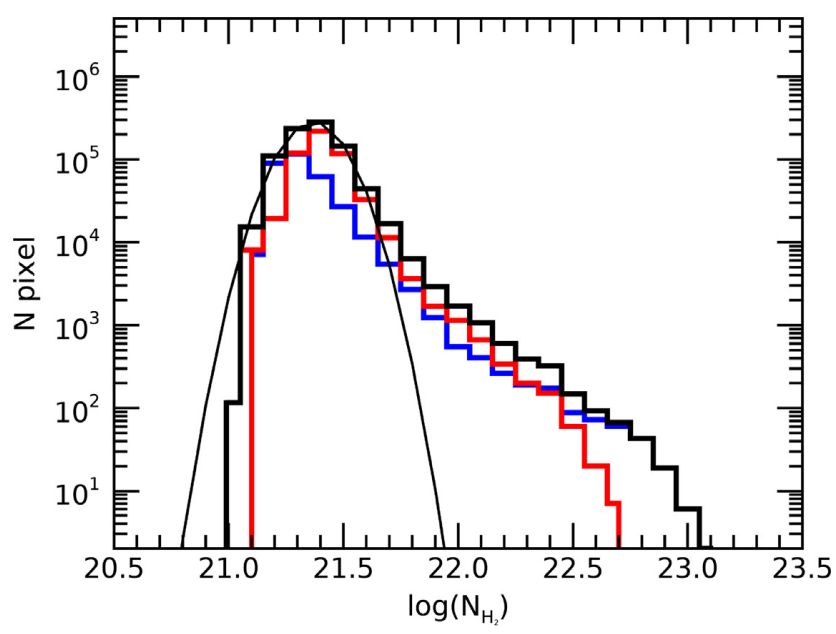

Figure 4. Histograms of column density from 18 arcsec column density map for the whole region (black). Note that the main contribution to the peak, which lies at $\sim 2.5 \times 10^{21} \mathrm{~cm}^{-2}$, is due to the Aquila East region (blue) while the peak of Serpens Main region (red) is shifted to lower column densities, $\sim 1.99 \times 10^{21} \mathrm{~cm}^{-2}$.

Table 2. Total mass of the entire Serpens region, Serpens Main, and East Aquila region computed from the high-resolution column density map (18.2 arcsec). The mass contained in contours of $A_{\mathrm{V}}>4$ and 7, respectively, is also reported.

\begin{tabular}{lccc}
\hline$M_{A_{V}}$ & $\begin{array}{c}\text { Serpens } \\
\left(\mathrm{M}_{\odot}\right)\end{array}$ & $\begin{array}{c}\text { Serp. Main } \\
\left(\mathrm{M}_{\odot}\right)\end{array}$ & $\begin{array}{c}\text { Aqu. East } \\
\left(\mathrm{M}_{\odot}\right)\end{array}$ \\
\hline $\mathrm{M}_{\mathrm{tot}}$ & 49400 & 8800 & 40500 \\
$\mathrm{M}_{4}$ & 11500 & 2900 & 8500 \\
$\mathrm{M}_{7}$ & 3000 & 1200 & 1800 \\
\hline
\end{tabular}

they insist on similar density ranges, which is not surprising as those regions are part of the same cloud complex (Ortiz-León et al. 2017).

\subsection{Mass and comparison with previous estimates}

Integrating the column density map over the entire region, we obtained a total Serpens mass of $\sim 49400 \mathrm{M}_{\odot}$ (see Table 2). The two main sources of error on the mass estimate are the background patches which typically affect the HGBS maps (4 per cent, see e.g. Könyves et al. 2015), and the error on the distance ( $\sim 11$ per cent). Therefore, by propagating these errors we obtain that the uncertainty on the mass estimate is about 12 per cent. However, the main source of uncertainty is the one in the dust opacity, which is only known to within $\sim 50$ per cent in the bulk of the target field (see Section 3.1). At very low column densities $\left(A_{v}<1-2\right)$, our adopted $\kappa_{0}$ value may depart from the 'true' $\kappa$ by more than 50 per cent (see Könyves et al. 2020; Di Francesco et al. 2020; Pezzuto et al. 2020). Therefore, we would quote an uncertainty of 50 per cent for the total Serpens mass. Just $\sim 6$ per cent of the mass is contained in regions with $A_{V}>7$, roughly considered a transition for the formation of prestellar cores (e.g. André et al. 2010; Könyves et al. 2015), if $N_{\mathrm{H}_{2}} / A_{\mathrm{V}}=9.4 \times 10^{20} \mathrm{~cm}^{-2}$ and $R_{\mathrm{V}}=3.1$ are assumed (Bohlin, Savage \& Drake 1978). Recently, Nakamura et al. (2017) studied the Serpens region in the ${ }^{12} \mathrm{CO}(J=2-1)$ and ${ }^{13} \mathrm{CO}(J=2-1)$. If we sum the mass of all the clouds analysed by Nakamura et al. (2017) located in the HGBS Serpens region and rescale to our assumed distance of 420 pc for Serpens Main and 484 pc for Aquila East, we obtain $23200 \mathrm{M}_{\odot}, \sim 47$ per cent of the mass we obtain.
Table 3. Mass estimates of the Serpens Main sub-region available in the previous literature. Masses in column 2 were derived at distances in column 1, and through the tracer(s) in column 3 by authors in column 4 . In column 5 , these masses are rescaled to the distance of $420 \mathrm{pc}$ adopted in this paper.

\begin{tabular}{lrccr}
\hline $\begin{array}{l}d \\
(\mathrm{pc})\end{array}$ & $\begin{array}{l}M_{\text {orig }} \\
\left(\mathrm{M}_{\odot}\right)\end{array}$ & tracer & Reference & \multicolumn{1}{c}{$\begin{array}{c}M_{420} \\
\left(\mathrm{M}_{\odot}\right)\end{array}$} \\
\hline 220 & 250 & $\mathrm{C}^{18} \mathrm{O}(J=1-0)$ & McMullin et al. (2000) & 911 \\
310 & 300 & $\mathrm{C}^{18} \mathrm{O}(J=1-0)$ & Olmi \& Testi (2002) & 550 \\
311 & $>1450$ & $\mathrm{C}^{18} \mathrm{O}(J=2-1)$ & White et al. (1995) & $>2644$ \\
& $>1450$ & $\mathrm{C}^{17} \mathrm{O}(J=2-1)$ & White et al. (1995) & $>2644$ \\
415 & 3213 & $N_{\mathrm{H}_{2}}$ & Roccatagliata et al. (2015) & 3290 \\
\hline
\end{tabular}

To compare our estimate to previous works on Serpens Main, we considered only the Serpens region analysed in the previous literature (from $\sim 250 \mathrm{M}_{\odot}$ to $\sim 4000 \mathrm{M}_{\odot}$ ), which is indicated in Fig. 1 . We derived a total mass of $8844 \mathrm{M}_{\odot}$ for Serpens Main.

As shown in Table 3, mass estimates of Serpens Main have been computed in the literature by adopting different distances and tracers. The disagreement (10-30 per cent) between our result and the works of McMullin et al. (2000), Olmi \& Testi (2002), and White, Casali $\&$ Eiroa (1995) might be explained by the different tracers used for estimating the mass. Using the $\mathrm{C}^{18} \mathrm{O}$ transition line, the contribution of the diffuse gas is not detected.

Thus, we found overall higher mass estimates for the Serpens Main region with respect to previous works. This difference is mainly due to the method we use to compute the sub-region mass. Indeed, we estimate the mass from the column density, while other works are based on spectroscopic tracers, which could underestimate the mass value. However, we cannot exclude we are overestimating the total mass of the cloud. Indeed, the Aquila East region is very close to the Galactic plane, and the global background emission is large (decreasing away from the Galactic plane), this may end up with large values of the cloud mass, which is affected by the emission uncorrelated with the cloud we are studying. On the contrary, when analysing molecular line data, e.g. CO data, since there is an information on the velocity, only the emission corresponding to the cloud, at the velocity of the cloud, is taken into account, thus the masses are lower. Moreover, we observed the dust emission which is present even in the diffuse gas in contrast to the $\mathrm{CO}$ which is not formed below a few $100 \mathrm{~cm}^{3}$. Our estimates are therefore more complete and it is expected we get larger masses then.

A more direct comparison of the cloud mass estimation is possible with Roccatagliata et al. (2015), who used the same Herschel data we use in this paper, but only for the sub-region we refer to as Serpens Main. They reported a total mass of this sub-region 2.7 times smaller than the estimate we found (see Table 3). Comparing the column density histograms, we find a global disagreement of a factor of $\sim 4$ (compare our Fig. 4 and fig. 4 of Roccatagliata et al. 2015). Moreover, Roccatagliata et al. (2015) assumed a different dust opacity law (Ossenkopf \& Henning 1994) with $\beta=1.9$, implying a dust opacity coefficient about $\sim 2$ times higher than ours, more appropriate for denser protostellar cores than for prestellar cores. The choices on the dust opacity assumptions should account for most of the difference in the derived masses. This difference can be understood, in turn, from the different temperatures found in the sub-region. Indeed, systematically higher temperatures typically lead to lower column densities. Roccatagliata et al. (2015) claimed a minimum temperature of $19 \mathrm{~K}$ and an average temperature of about $24 \mathrm{~K}$, while our values are lower, as discussed above. Moreover, these authors included $70 \mu \mathrm{m}$ emission in their modified blackbody 
fits, while, as said above, we do not. Note also that Roccatagliata et al. (2015) acknowledged that they found a mass 7 times lower than White et al. (1995) lower limit. On the contrary, our analysis satisfies this independent lower limit.

\section{SOURCE DETECTION AND CLASSIFICATION}

A dense core is a roundish structure that can be considered as the final result of cloud fragmentation, and whose gravitational collapse could give rise to a star or a multiple star system. Based on this concept, we generate a catalogue of starless cores for all the star-forming regions of the Gould Belt observed by Herschel. To ensure uniformity with other HGBS works, this catalogue is built following the procedure already described by Könyves et al. (2015) and Marsh et al. (2016) as a two-step process:

Step 1: Detection. Source extraction is carried out by the multiwavelength, multiscale software getsources (Men'shchikov et al. 2012) that simultaneously analyses the Herschel images between 70 and $500 \mu \mathrm{m}$, as well as the 'high-resolution column density' and the 'temperature corrected' maps (Section 3), producing a preliminary version of a core catalogue.

A complete summary of the source extraction method can be found in section 4.4 of Könyves et al. (2015), and full technical details are provided in Men'shchikov et al. (2012). After the extraction, properties of detected sources are measured in the original observed images at each wavelength (Könyves et al. 2015). For producing HGBS first-generation catalogues of starless and protostellar cores, two sets of dedicated getsources extractions are performed, optimized for the detection of dense cores and YSOs/protostars, respectively. In the former, the $160,250,350$, and $500 \mu \mathrm{m}$ maps, and the high-resolution column density image are considered together in a multiwavelength approach. In particular, the $160 \mu \mathrm{m}$ component of the detection image is 'temperature-corrected' to reduce the effects of strong, anisotropic temperature gradients present in parts of the observed fields.

A second set of getsources source extractions is performed to trace the presence of YSOs/protostars. In this process, at the detection stage only the PACS $70 \mu \mathrm{m}$ data are used. Point-like $70 \mu \mathrm{m}$ emission properly traces the internal luminosity, and therefore the presence itself, of a protostar (e.g. Dunham et al. 2008). We eliminated contamination due to galaxies and other non-YSOs by looking for those sources in Simbad database within 2 arcsec from the centroid coordinates. At the end of this process, we count 124 of those objects, named protostellar cores. Although protostellar cores are contained in the catalogue, they are not analysed in this work, but will be discussed separately in a subsequent paper.

Step 2: Classification. The raw source list outputted by getsources in the region studied here is then filtered to select only dense starless cores, by applying the following criteria:

(i) Column density detection significance higher than 5 in the highresolution column density map, where significance refers to detection at the relevant single spatial scale, as defined by Men'shchikov et al. (2012);

(ii) High-resolution column density signal-to-noise ratio at the peak of the emission, $(\mathrm{S} / \mathrm{N})_{\text {peak }}>1$;

(iii) Global detection significance over all wavelengths (Men'shchikov et al. 2012) greater than 10;

(iv) Significance of flux detection greater than 5 for at least two wavelengths between 160 and $500 \mu \mathrm{m}$; (v) Flux measurement with $\mathrm{S} / \mathrm{N}>1$ and monochromatic detection significance greater than 5 for at least one band between 160 and $500 \mu \mathrm{m}$.

(vi) $70 \mu \mathrm{m}$ peak intensity per pixel less than 0 , which is typical of noise-dominated areas, or detection significance less than 5 for this band, or source size at this band larger than 1.5 times the band (at this stage, namely the last one based on constraints on detection parameters, the candidate sources remain 1081);

(vii) The source is not spatially coincident within 6 arcsec of a known IR source of at least one of the following catalogues: Spitzer Space Telescope (SST; 137 matches found), Widefield Infrared Survey Explorer (WISE; 149 matches found) and Simbad Database (6 matches found) for further infrared observations;

(viii) The source is not spatially coincident within 6 arcsec with a known galaxy, i.e. a comparison with NASA Extragalactic Database (NED) found no matches;

(ix) The source appears real on visual inspection of the images between 160 and $500 \mu \mathrm{m}$. In this respect, 15 per cent of the dense starless sources were visually rejected.

The resulting 833 starless cores are contained in the catalogue available in the online version of this paper, and from the HGBS website (see the footnote 2 in Section 2). In this website, all the maps discussed in this paper are also available. The catalogue consists of reports for each source, see Tables A1 and A2 in Appendix A.

Fig. 1 shows the column density map, in which we notice a largescale gradient which corresponds to that seen in the temperature map (see Section 3), although less evident. On this column density is plotted the distribution of the 833 sources of the getsources catalogue we obtained after Classification step. We are confident that all 833 detected cores (starless and protostellar) of our census are robust, based on visual inspection checks.

\subsection{Estimation of core masses and temperatures}

Similarly to the approach described in Section 3, the core masses and the temperatures can be estimated by fitting the modified blackbody function (equation 2) to their SED obtained through the getsources software. In the case of temperature and column density maps, the SEDs are composed by single and unsubtracted pixel fluxes, while in the case of source fluxes they are background-subtracted and integrated over the entire source area. This different treatment of the background, which changes with wavelength, can lead to different estimates of core temperature and mass, whether they are derived from the source SED, or taken from temperature and column density maps (cf. e.g. Kirk et al. 2013).

Furthermore, we notice that actually the structure of a quiescent core might be more accurately modelled as a series of dust layers, the outermost of which is the warmest, heated by external radiation, and the innermost the coldest (Roy et al. 2014). This is the reason why 'observed' core masses, obtained from modified blackbody fit, can be typically $\sim 50$ per cent lower than true core masses, since SED-derived temperatures tend to overestimate the mass-averaged temperatures of starless cores (Könyves et al. 2015). Anyway, modified blackbody fit remains a reasonable and useful way to study mass and temperature of each single core.

The masses were derived assuming $d=484 \mathrm{pc}$ for the Aquila East region, and $d=420 \mathrm{pc}$ for the Serpens Main region. The physical core radius is obtained from the angular FWHM estimated by getsources in the high-resolution (18.2 arcsec) column density map. In our catalog (see Table A2), we provide estimates for both the deconvolved and the observed radius of each core (estimated as the 
geometrical average between the major and minor FWHM sizes). For each core, the peak column density, average column density, central-beam volume density, and the average volume density were then derived based on their mass and radius estimates (see section 4.6 in Könyves et al. 2015 for details). In Table A2, all these observables are listed for the whole sample of selected Herschel cores. For SEDs composed by only one or two significant flux measurements (see appendix A in Pezzuto et al. 2020), the fitting procedure was not applied. We also did not look for the best fit in cases where $F_{v}(350 \mu \mathrm{m})<F_{\nu}(500 \mu \mathrm{m})$. For all these cases, we adopt a fiducial temperature equal to the median temperature of all the reliable SEDs, excluding the protostars. The uncertainty of this median is arbitrarily large (the median $\Delta T / T$ for reliable sources is $\sim 3$ per cent). For these sources, which are the $\sim 20$ per cent of the total starless cores, the mass was computed from the measured integrated flux density at the longest significant wavelength, assuming the fiducial temperature.

We calculated the completeness limit using the same model approach as described in appendix B.2 of Könyves et al. (2015). In this way, the $\sim 80$ per cent completeness level is estimated to be at $\sim 0.8 \mathrm{M}_{\odot}$ in true prestellar core mass.

To identify dense cores candidates to likely form new stars, one has to verify whether sources are gravitationally bound. A selfgravitating core satisfies the virial condition:

$\alpha_{\mathrm{vir}}=M_{\mathrm{vir}} / M_{\mathrm{obs}} \leq 2$

(Könyves et al. 2015). The virial mass $\left(M_{\text {vir }}\right)$ could be computed by measuring the mean velocity dispersion spectroscopically, but such observations are not available for all the sources with a spatial resolution comparable to the Herschel one. Therefore, we use the Bonnor-Ebert (BE) criterion to select self-gravitating objects. According to this criterion, a core whose mass exceeds the BE critical mass will collapse (Bonnor 1956). A BE sphere, whose outer radius is $R_{\mathrm{BE}}$, has a critical mass given by

$M_{\mathrm{BE}}=2.4 R_{\mathrm{BE}} a_{s}^{2} / G$,

where $G$ is the gravitational constant and $a_{s}^{2}=k T / \mu m_{H}$ is the square of the sound speed, with $k$ being the Boltzmann's constant, $T$ is the temperature, and $\mu=2.33$. We computed the BE mass starting from the deconvolved core radius $R_{\text {deconv }}$ measured as the geometric mean of the source FWHM major and minor axes as estimated by getsources from the high-resolution column density map, after beam deconvolution, and by using as gas temperature the dust temperature estimated through the SED fit. In this way, we find that 455 starless cores are robust prestellar cores, i.e. with masses larger than $M_{\mathrm{BE}} / 2$, where the factor $1 / 2$ follows the analogy with the criterion of cloud collapse based on the virial condition (equation 6). Könyves et al. (2015) show that if they classify as prestellar a core for which $\alpha_{\mathrm{BE}}=$ $M_{\mathrm{BE}} / M_{o b s} \leq 2$, Monte Carlo simulations performed to assess the mass completeness of the survey suggest that this criterion may be too restrictive. Indeed, it selects only $\sim 85$ per cent of the simulated $\mathrm{BE}$ cores detected by getsources after the source classification. Thus, they proposed a less demanding and size-limiting criterion, based on the results of Monte Carlo simulations, which produce an envelope containing $>95$ per cent of the simulated BE cores after getsources extraction:

$\alpha_{\mathrm{BE}} \leq 5 \times\left(\mathrm{HPBW}_{\mathrm{N}_{\mathrm{H}_{2}}} / \mathrm{FWHM}_{\mathrm{N}_{\mathrm{H}_{2}}}\right)^{0.4}$

where $\mathrm{HPBW}_{\mathrm{N}_{\mathrm{H}_{2}}}=18.2^{\prime \prime}$ is the resolution of the high-resolution column density map, and $\mathrm{FWHM}_{\mathrm{N}_{\mathrm{H}_{2}}}$ is the measured FWHM source diameter in the same map. It is important to stress that this is an empirical result found by getsources extractions on Herschel observations. Using equation (8), we obtained that 604 are prestellar
Table 4. Classification of the Serpens dense cores. After the checks described in Sections 4 and 4.1, in the getsources catalogue there are 833 dense cores, 709 of them are starless, and 124 of them are protostellar cores. 105 starless cores are gravitationally unbound cores, the remaining 604 gravitationally bound cores are candidate prestellar cores. Finally, 455 prestellar cores are classified as robust because their mass fulfils the BE criterion (see the text).

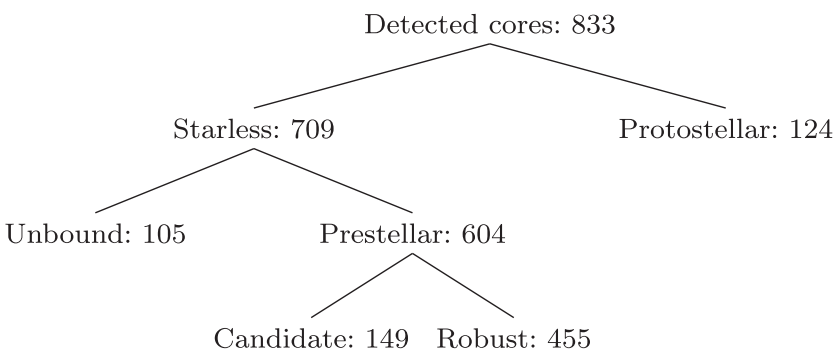

cores (i.e. 149 candidate cores are added to the robust ones). In Table 4, the classification scheme is summarized.

For the Serpens star-forming region, the percentage of prestellar cores over the overall starless ones is $\sim 85$ per cent, and the percentage of only robust cores is $\sim 64$ per cent. Note, however, that this fraction can be affected by a distance bias. In particular, the mass increases as the square of the distance assumed for the region, while the BE mass increases linearly. This implies that the aforementioned fractions generally increase at increasing distance and, as a borderline case, over a certain distance 100 per cent of sources would have a mass larger than the BE mass (cf. Elia et al. 2013). For this reason, comparisons between regions at distances significantly different based on this observable should be performed with caution.

In Fig. 5, we plot the core mass versus radius relation, which essentially confirms the low-mass nature of the star formation in Serpens/Aquila East region, in the light of Krumholz \& McKee (2008) and Kauffmann \& Pillai (2010) requirements for a core to host the formation of massive stars. In both these works, a powerlaw threshold for the $M$ versus $r$ relation is set, that we interrupt at the bottom at $24 \mathrm{M}_{\odot}$ assuming a minimum mass of $M>8 \mathrm{M}_{\odot}$ for a high-mass star and a typical core-to-star efficiency of $\varepsilon=1 / 3$ in the process. If we compare our results with the two thresholds, we find 2 and 3 objects, respectively, compatible with the possible formation of a high-mass star. However, in the following we compute a specific core-to-star efficiency for this region, finding $\varepsilon=0.2$ (see Section 5). Adopting this value, the lower limit for compatibility with high-mass star formation increases to $40 \mathrm{M}_{\odot}$, which is not fulfilled by any core in our sample. This figure also shows that our sample is spread not only in mass, as discussed above, but also in size, denoting a certain relation with the source type: robust bound cores sizes are contained between $0.01 \mathrm{pc}$ and $0.25 \mathrm{pc}$, candidate bound cores between 0.01 and $0.19 \mathrm{pc}$ (therefore, this is the most compact subset), and, finally, unbound core sizes between 0.01 and $0.21 \mathrm{pc}$. In Fig. 5, a few sources with size larger than $0.2 \mathrm{pc}$ are seen. Taking into account the classification of Bergin \& Tafalla (2007), Di Francesco et al. (2007), and André et al. (2014), such sources do not fulfil the definition of core, but rather that of clump, with an underlying but unresolved structure, probably containing multiple cores. Although the thresholds on which this classification is based should actually be considered loose, it is undeniable that the distance(s) assumed for the Serpens/Aquila East region makes it as a slightly particular case with respect to other, generally closer regions surveyed in the HGBS, in which the resolution-distance combination ensures that all detected compact sources are cores. As we can observe in Fig. 5, our 


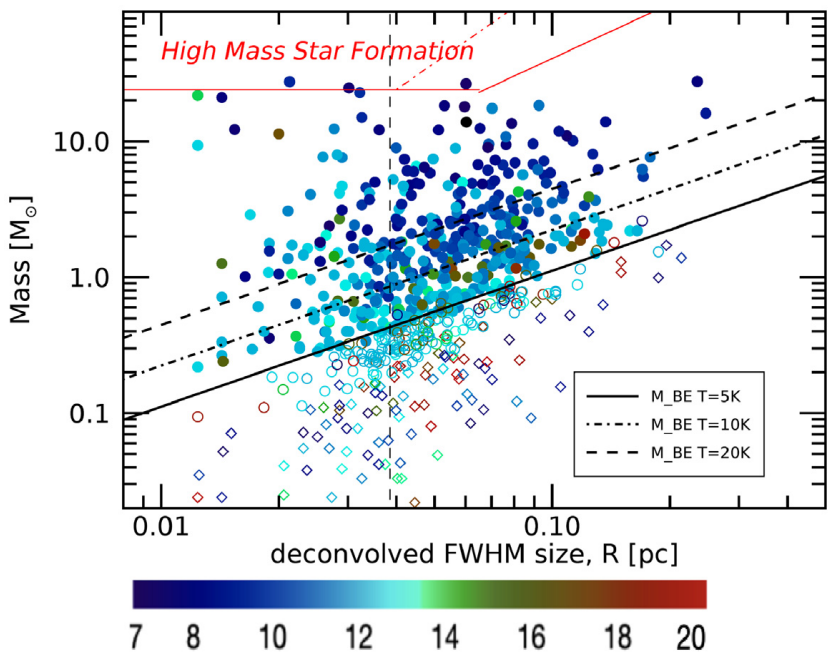

Figure 5. Mass versus size diagram for the starless cores. The vertical dashed line indicates the corresponding physical resolution at $d=484 \mathrm{pc}$. Robust prestellar cores are shown with filled circles, candidate prestellar cores with open circles, and unbound cores with open diamonds, respectively. The colour indicates the temperature of the source in $\mathrm{K}$, following the colour bar: from blue to red, the temperature linearly increases from 7 to $20 \mathrm{~K}$. The black lines represent the $\mathrm{BE}$ mass computed at different temperatures: the solid line at $5 \mathrm{~K}$, the dot-dashed one at $10 \mathrm{~K}$, and the dashed one at $20 \mathrm{~K}$. The robust cores are all located above the $5 \mathrm{~K}$ line, respectively. Red dashed and continuous lines are the Krumholz \& McKee (2008) and Kauffmann \& Pillai (2010) limits for high-mass star formation, respectively, the former being contained in the latter.

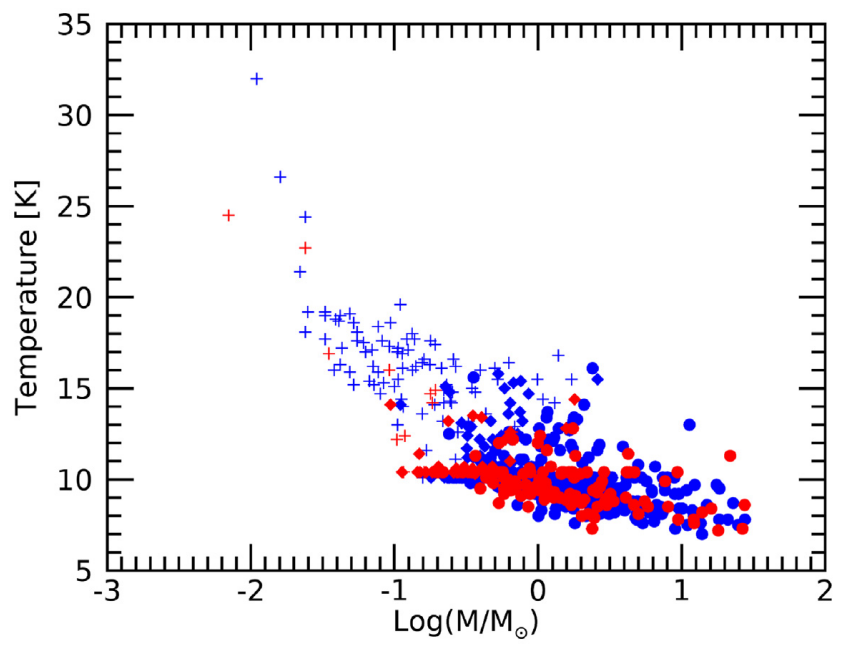

Figure 6. Core temperature versus core mass. Filled circles are robust prestellar cores, filled diamonds are tentative prestellar cores, and crosses are unbound cores. Cores situated in Aquila East and in Serpens Main are shown in blue and red.

analysis seems to verify that, as the star formation scenario predicts, the core future collapse depends only on mass and not on the size of the core. Indeed there is no correlation between the size range and the cores type. The Fig. 6 shows the plot of cores temperatures versus mass. We find a negative correlation between temperature and mass, as expected according to the scenario in which the dust is heated by the interstellar radiation field (Stamatellos, Whitworth \& Ward-Thompson 2007). The possibility that this is an artefact due to the analytic relation between these two quantities in the adopted

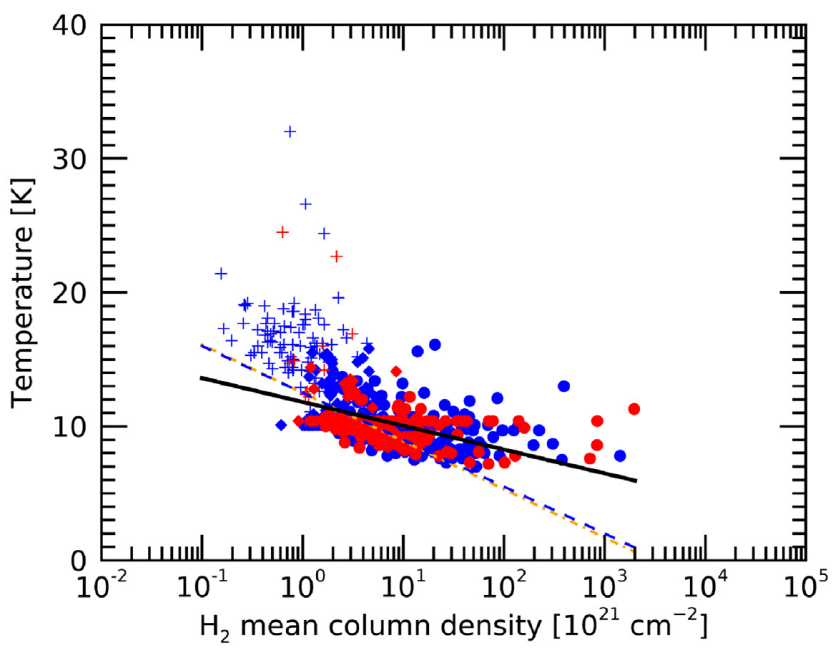

Figure 7. Mean dust temperature of cores plotted as a function of mean column density of cores, $N_{\mathrm{H}_{2}}$ diagram. Symbols are like in Fig. 6 . The blue dashed line is the power-law fit to data of Bresnahan et al. (2018), the pink dashed line is the fit to data of Marsh et al. (2016), and the black solid line is the relation found in this work.

model (modified blackbody) is significantly lower with respect to works based on monochromatic observations: here the five-band analysis in a wavelength range generally containing the peak of the core SED ensures an independent estimate for temperature and mass. Another source of bias in estimating the mass when using a singletemperature model is the core internal temperature gradient. These biases have been estimated to be typically at the 30 per cent level (see e.g. Könyves et al. 2015; Marsh et al. 2016), i.e. smaller compared to the correlation seen in Fig. 6. We thus conclude that this correlation is real. Moreover, as expected, unbound cores lie on the upper-left part of the diagram, while prestellar cores are found in the bottomright part. We note also that most massive cores are cold, whereas we cannot conclude that least massive cores are typically warm, because our sample is limited by the sensitivity of the instrument. This issue is widely discussed in appendix A of Marsh et al. (2016).

To remove any dependence on the distance, still implicit in Fig. 6, we also plotted the dust temperature versus the mean column density for the starless cores (Fig. 7). To allow a quantitative comparison with Marsh et al. (2016), we arbitrarily fit a power law to data

$T=a \log _{10} \frac{N_{\mathrm{H}_{2}}}{10^{21} \mathrm{~cm}^{-2}}+b$,

finding $a=-1.8 \pm 0.1$ and $b=11.8 \pm 0.1$. Similar relations, rescaled to units of $N_{\mathrm{H}_{2}} / 10^{21} \mathrm{~cm}^{-2}$ to allow comparison, were found for Taurus ( $a_{\text {Tau }}=-3.6$ and $b_{\text {Tau }}=12.5$, Marsh et al. 2016) and for Corona Australis $\left(a_{\mathrm{CorA}}=-3.5\right.$ and $b_{\mathrm{CorA}}=12.5$, Bresnahan et al. 2018). Marsh et al. (2016) reasonably posited that the coefficients of such fits are not expected to be universal, but depend on the intrinsic conditions of the regions. Considering the three regions for which this analysis was carried out so far, Corona Australis and Taurus show quite similar coefficients, while the ones we obtained in Serpens are different. Extending this analysis to further star forming regions it will be possible to depict a more defined scenario on the basis of a larger statistics.

\subsection{Spatial distribution of cores}

In the following, we analyse the spatial distribution of cores, in the light of the identified sub-classes, with the help of Fig. 8, and Table 5. 

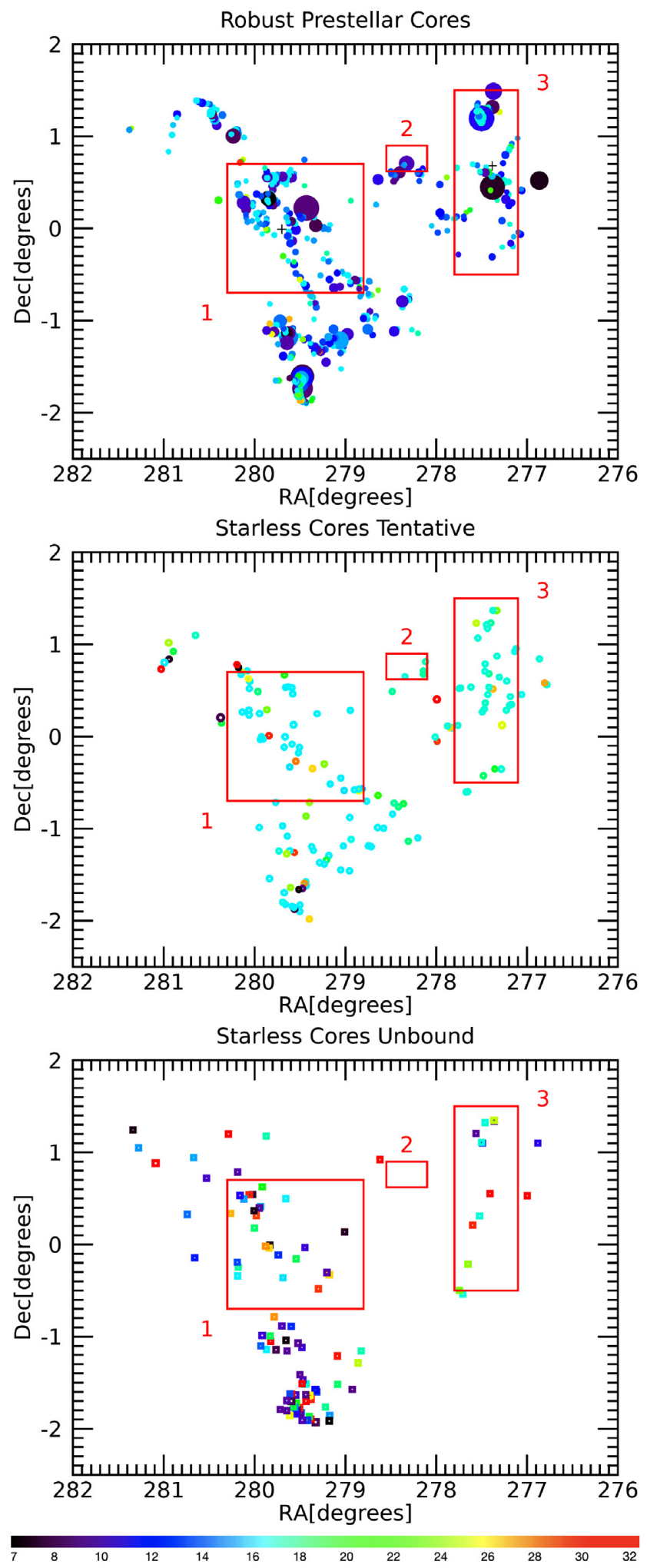

Figure 8. Spatial distribution of robust prestellar (top), candidate bound (middle), and unbound (bottom) cores, respectively. The colour, from black to red, represents the temperature from 7 to $32 \mathrm{~K}$, respectively. The size of the circles and the squares represents the mass, from 0.01 to $27.5 \mathrm{M}_{\odot}$.
Table 5. Average mass and temperature for each sample of cores. Prestellar cores are subdivided here in robust and candidate, cf. Table 4.

\begin{tabular}{lcc}
\hline Type of cores & $M\left(\mathrm{M}_{\odot}\right)$ & $T(\mathrm{~K})$ \\
\hline Robust prestellar cores & $2.93 \pm 0.35$ & $9.7 \pm 0.4$ \\
Candidate prestellar cores & $0.44 \pm 0.14$ & $11.0 \pm 0.8$ \\
Unbound cores & $0.20 \pm 0.02$ & $15.4 \pm 0.6$ \\
\hline
\end{tabular}

Mean values of mass and temperature for each sub-sample support the evidence, already found in previous HGBS works and clearly shown also in Figs 5 and 8, that more massive cores are generally the coldest, and this is verified for each of the three core classes. In general, robust bound cores are the most massive and coldest, while, on the other side, unbound cores are the least massive and warmest, with candidate bound prestellar cores lying in the middle between these two populations. This behaviour is strictly due to the modified blackbody function (equation 2) we use to obtain temperature and mass values, but note that the detection sensitivity cut-off does not allow us to know whether there are cold unbound cores, as discussed in Marsh et al. (2016).

The spatial distribution of candidate prestellar and unbound cores seems to be more widely distributed than that of robust bound prestellar cores, which follows instead the underlying filamentary structure (as deeply discussed in Section 7).

In the following, we discuss the spatial relation between prestellar cores and YSOs (see Figs 8 and 9). We consider the area observed by Spitzer and analysed by Winston et al. (2007), Harvey et al. (2007), and Dunham et al. (2015), in which the main clusters of YSOs in Serpens Main and Aquila East regions are located, and that is composed by three 'boxes' (shown in Fig. 9). These areas contain more massive robust bound cores (74 in box 1, 6 in box 2 , and 154 in box 3 , respectively), than candidate bound ( 22 in box 1 , 4 in box 2, and 39 in box 3, respectively), and unbound cores 11 in box 1,1 in box 2 , and 26 in box 3, respectively). In other words, about half of the prestellar cores population (43 per cent of the robust prestellar cores and 52 per cent of the candidate prestellar cores, 45 per cent of the prestellar cores in total, respectively), and about one-third of the unbound cores population (36 per cent) is located in these Spitzer regions. This indicates the presence of a further mass reservoir in the areas already hosting star formation. Moreover, the higher degree of prestellar core clustering in regions rich of YSOs suggests that the same event that caused the formation of YSOs in Serpens Main, also produced the condensation of matter in the cores of this sub-region, which became denser and more massive, and thus ready to collapse in case of a new triggering event, confirming similar considerations by Winston et al. (2007), Harvey et al. (2007), and Seale et al. (2012), based only on analysis of the spatial distribution of YSOs different classes. According to this scenario, the Spitzer sources spatially related with cores are the young embedded protostars (Class 0/I), while the sources on the border the more evolved pre-main sequence (PMS; i.e. Class II/III) YSOs.

However, looking at shorter scales, there is not strict spatial coincidence, as expected, between massive prestellar cores and YSOs. In Fig. 9, we can see that in box 1 one of the most massive cores (at the top of the panel) is closely surrounded by protostars and PMS stars in the expected way, while the other massive core (at the centre of the panel) appears to have only group of dispersed protostars around it. A similar configuration is seen in boxes 2 and 3, where the most massive cores are not related to clustered protostars. In fact, a closer spatial correlation with YSOs would 

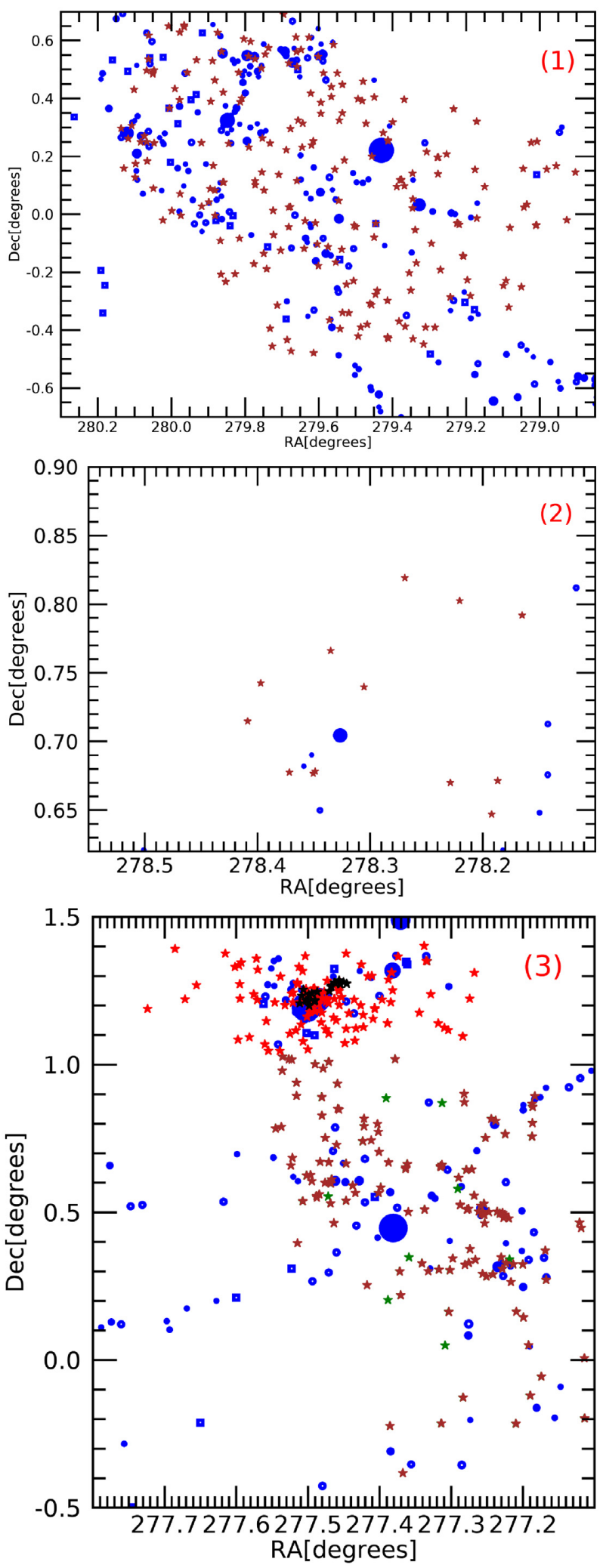

Figure 9. Spatial distribution of cores population (blue circles and squares) and YSOs listed in Winston et al. (2007) (protostars in black and PMS YSOs in red), in Harvey et al. (2007) (protostars in grey and PMS YSOs in green), and Dunham et al. (2015) (protostars in brown), respectively. Panels are the red rectangles \#1 (top), \#2 (middle), and \#3 (bottom) shown in Fig. 8, corresponding to the area observed by Spitzer. Notice that axes of each panel have different scales. be expected for protostellar cores, and this topic will be discussed in a subsequent paper focused on the Herschel protostellar cores population.

\section{THE PRESTELLAR CORE MASS FUNCTION}

We computed the CMF for starless cores (Fig. 10). The Serpens/Aquila East prestellar CMF is well fitted by a lognormal function at masses lower than $2 \mathrm{M}_{\odot}$, and by a power law at higher masses. The completeness limit effectively lies where the mass distributions of candidate versus prestellar CMFs start to differ, enabling us to locate the peak and discuss the power-law slope, but not the lognormal fit.

In a simple scenario in which each core produces one stellar system, core masses map into stellar masses with a core-to-star efficiency. Using as a reference the peak position of the Chabrier (2005) IMF for multiple systems, and comparing it with that of the $\mathrm{CMF}$, we estimate an efficiency of $\varepsilon=0.2$ both for the overall region and for Serpens Main only (see Section 6.1), lower than typical estimates for this quantity $(\varepsilon=0.25-0.40$, Alves, Lombardi \& Lada 2007; Enoch et al. 2008; André et al. 2010). However, we want to stress that the core-to-star efficiency computed as we did, strongly depends on the distance. In this case, the low efficiency might be seen as a consequence of the larger distance of Serpens/Aquila East region with respect to other nearby star-forming regions. For this reason, compact structures detected in the Serpens/Aquila East region are typically larger than those found in closer regions and, correspondingly, the CMF lies on a range of higher masses.

About the high-mass tail, we remove from the sample two prestellar cores whose size is larger than 0.2 pc because they probably are not resolved (see Section 4.1), and find that the best-fitting powerlaw slope is $\gamma=-2.05 \pm 0.34$, which is in agreement with both the Salpeter (1955) and Chabrier (2005) IMFs slopes within the errors, but also with the CO clump distribution by Kramer et al. (1998), $\gamma_{\mathrm{CO}}=-1.65 \pm 0.10$. Since other clouds observed in the HGBS have found similar consistencies, this is a further evidence that statistical mass distribution of main sequence low-mass stars directly depends on the one of the prestellar phase (Motte et al. 1998; Testi \& Sargent 1998; Offner et al. 2014; André et al. 2014), i.e. it directly results from the fragmentation process of the molecular cloud.

From Table 6, which summarizes results obtained so far for various HGBS regions, we note that the CMF slope strictly can vary from region to region, also depending on the dense core sample used to build the CMF. Indeed, Corona Australis (Bresnahan et al. 2018) and Taurus (Marsh et al. 2016) CMFs have power-law slopes that are more similar to the CO clump slope (Kramer et al. 1998). Noticeably, in these regions the lowest values for the fraction of prestellar cores are found (e.g. in the Taurus region less than 20 per cent of cores are gravitationally bound), or even only unbound cores are present (Corona Australis). In addition, the Lupus complex does not show a power-law trend at all (Benedettini et al. 2018), because the statistics is quite poor and the uncertainties on the mass estimate are large. On the contrary, regions in which the CMF is obtained by considering bound starless cores, including Serpens/Aquila East, have a steeper high-mass power-law slopes $\gamma$ comparable to each other, and are in agreement within the error, with the Salpeter IMF slope. However, the core statistics of Ophiucus, Taurus, Corona Australis and Lupus regions (Ladjelate et al. 2016; Marsh et al. 2016; Bresnahan et al. 2018; Benedettini et al. 2018, respectively) are still not large enough to allow robust statistical conclusions about the power-law tail of the prestellar CMF of these regions, even for the youngest star-forming region. 


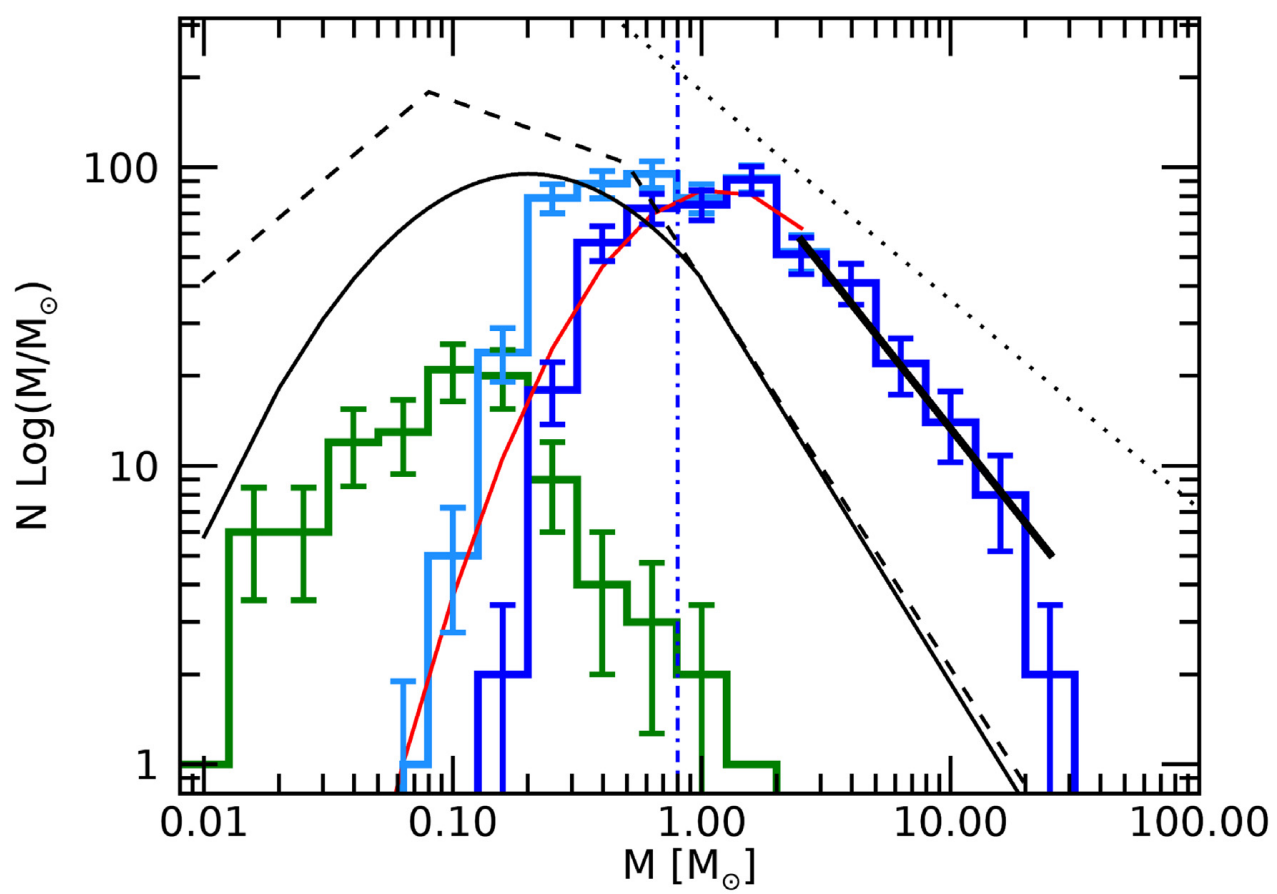

Figure 10. Core Mass Function (CMF) of Serpens/Aquila East star-forming region. The CMF of robust prestellar, candidate prestellar, and starless cores are shown as a blue, a light blue and a green solid histogram, respectively, with logarithmic bins. The prestellar CMF is well fitted by a lognormal function (red) up to 3-4 $\mathrm{M}_{\odot}$. At higher masses, the prestellar CMF shape follows a power law, with slope $-1.05 \pm 0.34$ (solid thick black line). For comparison, the CO clump mass function whose slope is $\xi_{\mathrm{CO}}=-0.65 \pm 0.10$ (Kramer et al. 1998), and the Kroupa (2001) and Chabrier (2005) stellar IMFs are plotted in dotted, dashed, and solid black, respectively. The Chabrier (2005) IMF is normalized to the peak of our CMF. The blue dot-dashed vertical line represents the mass completeness limit for prestellar sources in Serpens.

Table 6. CMF slope values $(\gamma)$, type of starless cores for which the CMF was computed, percentage of prestellar cores which lie on filamentary structure, distance in parsec and percentage of robust on starless cores $(r / s)$ for which the $\gamma$ is computed for the HGBS works published until now, respectively. The reported $\gamma$ slope of the last six clouds is referred to mostly bound cores, while for the first four it is referred to mostly unbound cores.

\begin{tabular}{|c|c|c|c|c|c|c|}
\hline Cloud & Cores type & $\gamma$ & On fil (per cent) & $d(\mathrm{pc})$ & $r / s$ (per cent) & Reference \\
\hline $\mathrm{CrA}$ & Mostly unbound & $-1.59 \pm 0.04$ & 88 & 130 & 14 & Bresnahan et al. (2018) \\
\hline Ophiuchus & Bound + unbound & No power law & 100 & 139 & 20 & Ladjelate et al. (2020) \\
\hline Taurus & Mostly unbound & $-1.55 \pm 0.07$ & 100 & 140 & 10 & Marsh et al. (2016) \\
\hline Lupus & Bound + unbound & No power law & 94 & $150-200$ & 11 & Benedettini et al. (2018) \\
\hline Aquila Main & Bound + unbound & $-2.33 \pm 0.06$ & 81 & 260 & 44 & Könyves et al. (2015) \\
\hline Perseus & Bound + unbound & $-2.30 \pm 0.06$ & 70 & 300 & 41 & Pezzuto et al. (2020) \\
\hline Cepheus Flare & Bound + unbound & $-2.40 \pm 0.14$ & 80 & 358 & 23 & $\begin{array}{l}\text { Di Francesco et al. } \\
\text { (subm.) }\end{array}$ \\
\hline Orion-B & Bound + unbound & $-2.27 \pm 0.24$ & 90 & 400 & 28 & Könyves et al. (2020) \\
\hline Orion-A & Bound + unbound & $-2.4 \pm 0.4$ & 67 & 414 & 77 & Polychroni et al. (2013) \\
\hline Serpens & Bound + unbound & $-2.05 \pm 0.34$ & 81 & $420-484$ & 64 & This work \\
\hline
\end{tabular}

Table 7. Statistics of cores detected in Serpens Main in the previous literature [TS98 = Testi \& Sargent (1998); E07 = Enoch et al. (2007)], compared to this work. The number of cores effectively used to compute the CMF is highlighted in boldface.

\begin{tabular}{lccc}
\hline & TS98 & E07 & This work \\
\hline Protostellar & 26 & 14 & 58 \\
Starless & 6 & 21 & 176 \\
(Unbound) & - & - & $(15)$ \\
(Prestellar) & - & - & $(\mathbf{1 6 1})$ \\
Total & $\mathbf{3 2}$ & $\mathbf{3 5}$ & 234 \\
\hline
\end{tabular}

The CMF shape estimation can be affected by the adopted binning (e.g. Olmi et al. 2013). A way to verify whether or not our choice of the bin size is reasonable is to consider the cumulative function of the CMF, which is not affected by this problem (cf. Testi \& Sargent 1998). Accordingly, we computed it for the prestellar cores population, as shown in Fig. 11. To check the high-mass powerlaw exponent, note that we computed the cumulative of the sample inversely ordered, i.e. by decreasing mass. This is because we want to check the power-law behaviour of the high-mass tail of the distribution. We compare this curve with the analytic cumulative 


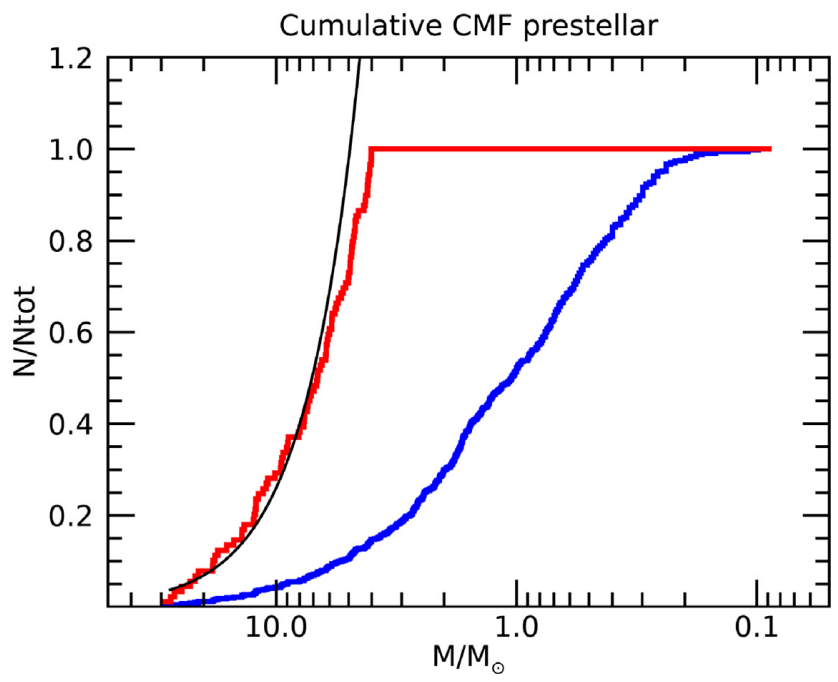

Figure 11. Inverse cumulative CMF for all prestellar cores (blue) and for those with $M>2 \mathrm{M}_{\odot}$ (red), with the cumulative of power-law best fit shown in Fig. 10 (black).

of the following power law:

$$
\frac{\mathrm{d} N}{\mathrm{~d} \log M} \propto M^{-\xi}
$$

obtained by fitting the CMF, where $\xi=\gamma-1 .^{3}$ In Fig. 11, we notice that the CMF shows a deficit of sources for $M>20 \mathrm{M}_{\odot}$. This could be a consequence of the presence of a mass reservoir larger than usually found in a low-mass star-forming region (Nakamura et al. 2017), suggesting that in Serpens/Aquila East the mass has accreted on to the most massive sources instead of the least ones. This could be explained by the fact that the more massive is the core, the largest (and quicker) is the mass accretion rate on to that source, as we know from high-mass star formation.

With respect to the previous analysis, HGBS results are more solid due to larger samples and lower limits in mass completeness. That was achieved thanks to the unprecedented sensitivity and angular resolution of Herschel. This enables us to probe not only the highmass part of the CMF but also the presence of a peak CMF mass and, in certain cases (Polychroni et al. 2013; Könyves et al. 2015), a significant part of the lognormal shape in the low-mass range. This is not the case of Serpens/Aquila East region, being the completeness limit almost coincident with the peak of the prestellar CMF. Albeit, we confirm the lognormal+power-law shape of the starless and prestellar CMFs. Moreover, there are 348 prestellar cores above the completeness limit, and 247 above the peak, which represents a significant improvement of the statistics over previous studies (Testi \& Sargent 1998; Enoch et al. 2007).

\section{FOCUS ON THE TWO SUBREGIONS}

In previous sections, we analysed the sample of cores extracted from the whole area of the sky surveyed with Herschel, but there are at least two reasons to discuss separately the core populations of Serpens Main and Aquila East, as identified in Fig. 1. First, the analysis contained in previous sections already highlighted some

\footnotetext{
${ }^{3}$ Note that in Figs 10, 13, and 14 the CMFs are plotted with respect $\mathrm{d} \log M$; therefore, the slope shown is $\xi$. To be compared with the values presented in literature one must derive $\gamma=\xi+1$.
}

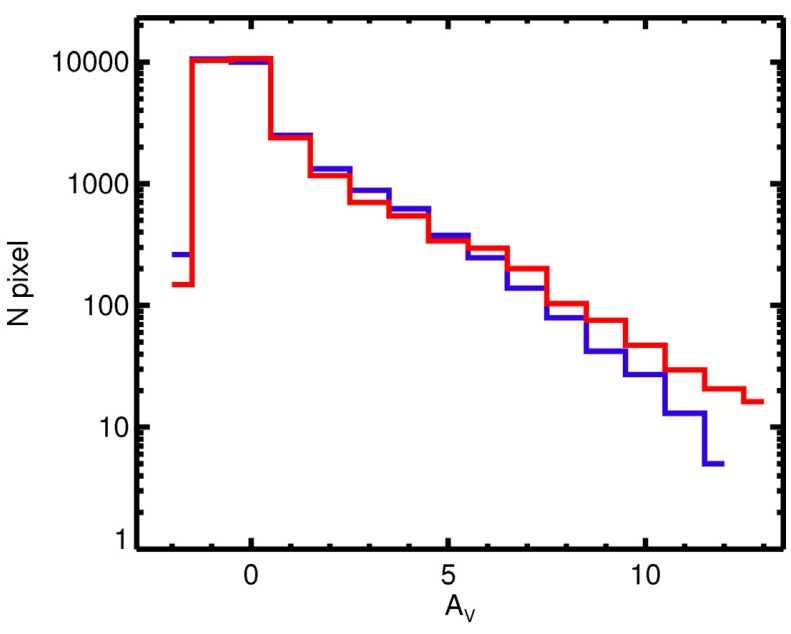

Figure 12. Histograms of visual extinctions estimated by Dobashi (2011) in the areas corresponding to our definition of Aquila East (blue) and Serpens Main (red). The latter histogram is normalized it by the total number of pixels involved in the former. Notice that the Dobashi (2011) maps contain negative values, which are responsible for the unrealistic bins before or at $A_{V}=0$.

differences between the two sub-regions (e.g. the presence of larger cores in the Serpens Main sub-region, together with a higher fraction of bound cores, see Fig. 8). Secondly, Serpens Main is by far the more deeply studied of the two sub-regions, thus, considering only the samples of cores from Serpens Main makes possible more direct comparisons with previous literature. In this section, we therefore retrace the analyses made in Sections 3, 4, and 5 in light of a comparison of the two sub-regions.

With Fig. 3, we already highlighted differences between histograms of both temperature and column density for the two subregions. The former is left-skewed for Aquila East, and peaks at higher temperatures compared to that of Serpens Main. Correspondingly, larger column densities are found in the Serpens Main subregion (Fig. 4). A similar reciprocal behaviour can be recognized in the histograms of visual extinction obtained from the $A_{V}$ maps of Dobashi (2011), shown in Fig. 12. This behaviour can be easily correlated with the aforementioned presence of the most massive cores in Clusters A and B of Serpens Main, and a lower rate of unbound cores, compared with Aquila East (Fig. 8). Higher temperatures (on average) in starless cores are generally explained by a more intense local interstellar radiation field (Evans et al. 2001). In the behaviour of dense cores temperature versus mean column density of cores (Fig. 7) and mass (Fig. 6), it is possible to note further differences between the two regions. First, the spread in mean column density and mass is smaller for the Serpens Main region than for the Aquila East one. This difference is due not only to the larger number of cores in the Aquila East, but also to the different composition of the two sub-populations. Indeed, Serpens Main starless cores are 176 in total, 15 (8 per cent) of which are unbound cores, and 161 (92 per cent) are prestellar cores (111 of which are in turn robust prestellar cores). In comparison Aquila East starless cores are 533 in total, 90 (17 per cent) of which are unbound cores and 443 ( 83 per cent) are prestellar cores, (344 of which are in turn robust prestellar cores). Thus, the Serpens Main sub-region has higher percentage of prestellar cores than Aquila East, further indicating the former as a more active star-forming region than the latter. Besides, the distribution of the Serpens/Aquila East population in Fig. 6 is flattened at the instrument sensitivity limit, suggesting 


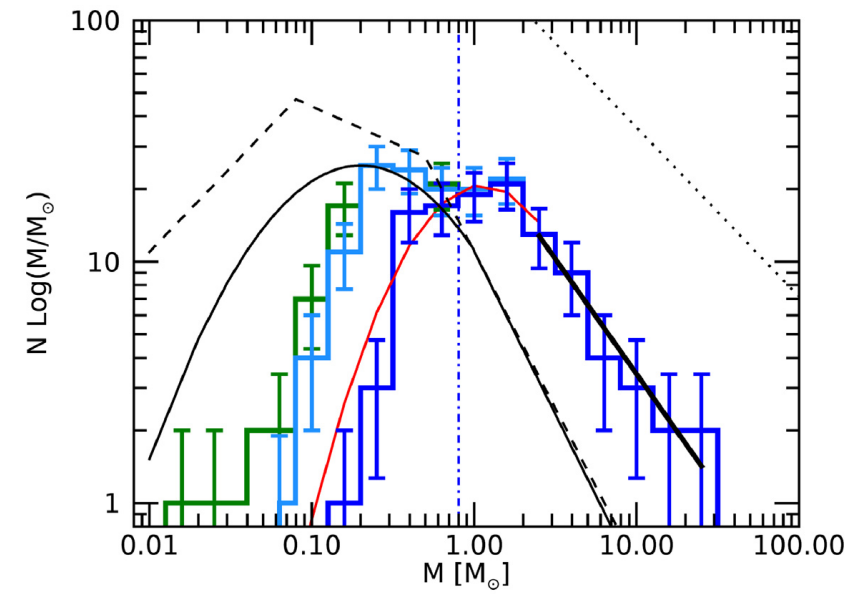

Figure 13. The same as Fig. 10, but for the Serpens Main region only. The high-mass tail of the prestellar CMF shape follows a power law, with slope $\xi_{\text {SM }}=-0.95 \pm 0.58$ (solid thick black line). One source with size larger than $0.2 \mathrm{pc}$ is ruled out from the sample.

that a significant percentage of Serpens/Aquila East cores might be less massive and colder but not detected, thus our results on mass and temperature could be overestimated. However, we cannot exclude that this difference in the amount of irradiation between Serpens Main and Aquila East might affect the core detection and extraction, which has been performed with a single and overall set-up for getsources .

\subsection{Serpens Main CMF}

It is interesting to analyse the CMF obtained only for the Serpens Main sub-region, since, as said before, it is directly comparable with previous works, although based on a much larger statistics.

The Serpens Main CMF (Fig. 13) shows a nearly lognormal behaviour up to $\sim 1-2 \mathrm{M}_{\odot}$, and a power-law trend at higher masses. The estimated slope of the power-law fit is $\gamma_{S M}=-1.95 \pm 0.58$, which is in agreement with that found for the entire region $(\gamma=$ $-2.05 \pm 0.34$, Section 5), although associated with a large error bar. In particular, the power-law fit fails in reproducing the rightmost bin.

To compare the CMF calculated for Serpens Main here and by Testi \& Sargent (1998) and Enoch et al. (2007), we note that the way we built our CMF differs in some aspects from those of the earlier studies. First, a larger statistics is involved in our case, due to better sensitivity of Herschel observations. Secondly, here the CMF is coherently built only with prestellar cores, while 14 out of 35 cores observed by Enoch et al. (2007) and 26 out of the 32 observed by Testi \& Sargent (1998) are protostellar cores, therefore not directly comparable with our result.

However, due to the large error, the slope of the power-law fit for the Serpens Main region is intermediate between the IMF and the CO clump mass function slopes. Probably, this might be interpreted as a statistical effect: the Serpens Main corresponds to a particular sub-region in which a concentration of relatively high-mass cores is present, but to recover a good fit of the typical power-law CMF it is necessary to complete the statistical basis with the masses from the remaining portion of the region.

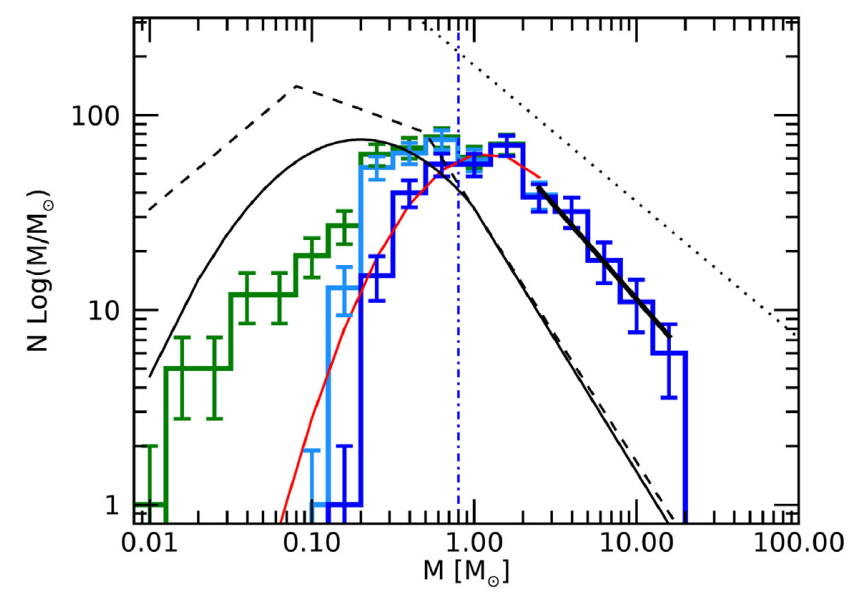

Figure 14. The same as Fig. 10, but for the Aquila East region only. The high-mass tail of the prestellar CMF shape follows a power law, with slope $\xi_{A E}=-0.95 \pm 0.41$ (solid thick black line). One source with size larger than $0.2 \mathrm{pc}$ is ruled out from the sample.

\subsection{Aquila East CMF}

The CMF of the Aquila East sub-region (Fig. 14) is obtained here for the first time. As the CMF of the Serpens Main sub-region, it shows a nearly lognormal behaviour up to $\sim 1-2 \mathrm{M}_{\odot}$, and a powerlaw trend at higher masses. The estimated slope of the power-law fit is $\gamma_{A E}=-1.95 \pm 0.41$, which is the same as the Serpens Main one, but with a smaller error bar (see Section 5), and is also in agreement with that found for the entire region $(\gamma=-2.05 \pm 0.34$, Section 5). Therefore, the comparison with other Gould Belt regions in Section 5 also applies to Aquila East.

The right tail of this CMF is closer to a power law with respect to that of Serpens Main, as indicated by the smaller uncertainty on the slope, which is probably related to the larger statistics: 443 prestellar cores are present in the Aquila East region, compared to 161 in the Serpens Main region.

Finally, we notice that also in the case of the Aquila East CMF, the slope of the power-law fit is intermediate between the IMF and the CO clump mass function slopes.

\section{RELATIONSHIP BETWEEN CORES AND FILAMENTARY STRUCTURE IN SERPENS}

André et al. (2010), André et al. (2014), and Könyves et al. (2015) described the relationship observed in Aquila Main between supercritical filaments and prestellar cores. The fact that most prestellar cores were seen to be along supercritical filaments and, on the contrary, unbound cores were found to be situated along subcritical filaments, suggests that star formation predominantly occurs in filaments, making them a key structure in the global star formation scenario. Although we do not include a specific filament analysis here, we want to address this topic by briefly discussing the spatial association between cores and filaments in the overall Serpens/Aquila East region.

To detect filaments in the Serpens/Aquila East data, we applied to the column density map the algorithm presented in Schisano et al. (2014) and Schisano et al. (2020), to which we refer the reader for a complete description of the algorithm. Here, we just summarize basic details. Among several definitions of filaments, given in the recent literature, the one adopted by Schisano et al. (2020) is 'any extended two-dimensional, cylindrical-like feature that is elongated and shows 


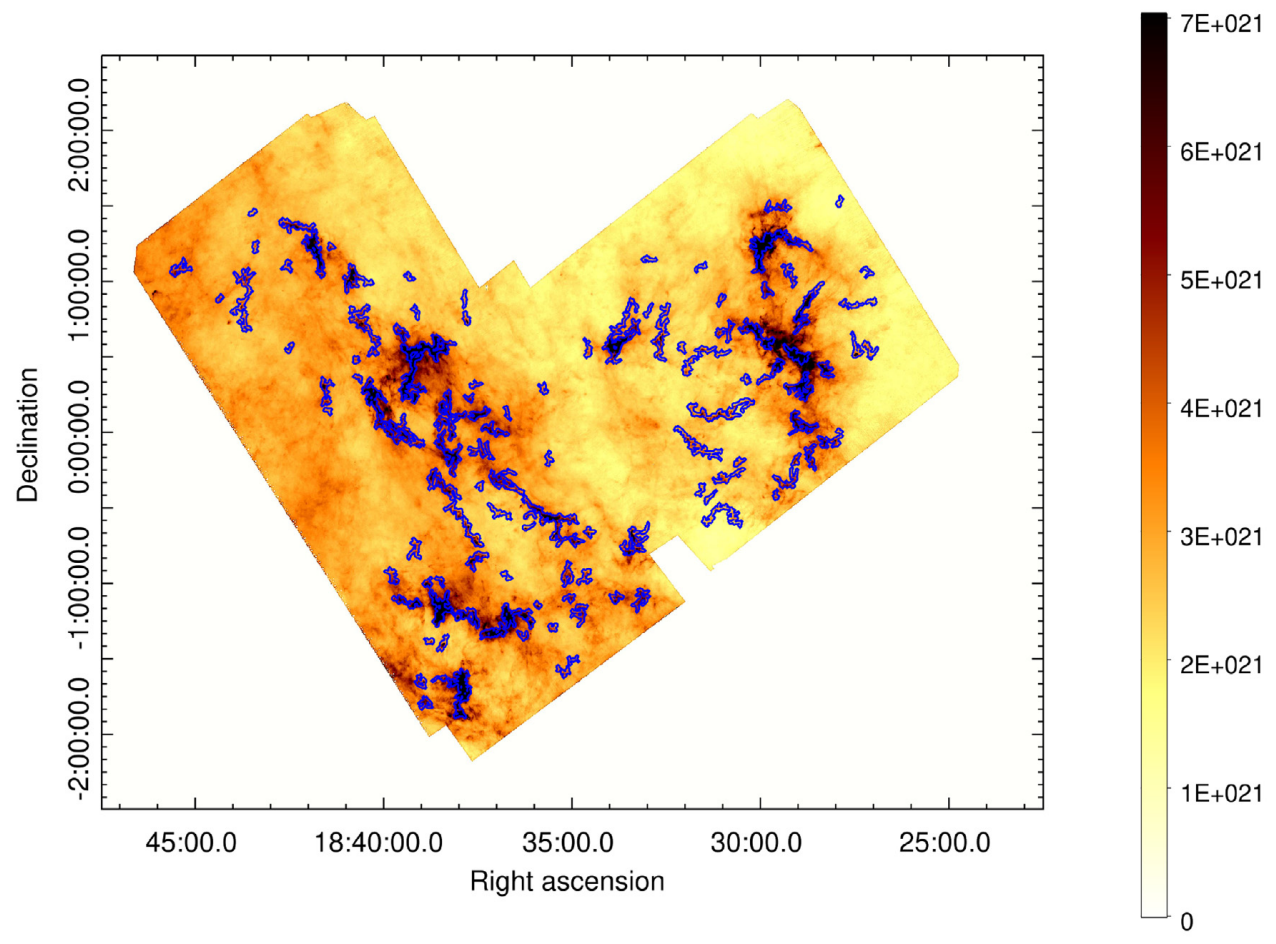

Figure 15. Boundaries of filamentary structures extracted as described in the text, overplotted on the column density map. The error bar displays values in $\mathrm{cm}^{-2}$.

a higher brightness contrast with respect to its surroundings'. Starting from this definition, such structures are identified by using the Hessian Matrix $H(x, y)$ of an image, its eigenvalues $\lambda_{a}, \lambda_{b}$, and their linear combination. The algorithm is applied to the column density map $N_{\mathrm{H}_{2}}(x, y)$. During the filament extraction phase, $H(x, y)$ is diagonalized and its eigenvalues computed. The diagonalization of the Hessian Matrix corresponds to the rotation of the axes towards the direction in which $N_{\mathrm{H}_{2}}(x, y)$ has the maximum and minimum variation. These variations are measured by $\lambda_{a}$ and $\lambda_{b}$, respectively. In this way, it is possible to properly identify a cylindrical shape.

This algorithm requests two input parameters: a threshold value $\operatorname{Tr}$ and a dilation parameter that permits to extend the initial mask (essentially based on the filament spine) in order to include the entire filament area with its borders. In appendix B of Schisano et al. (2020) the range of variability for these parameters is widely investigated, and the choice of default values for them is justified. Here we use the default value to set the dilation parameter, and $T r=3.5 \times \sigma$ as threshold value, different from the default value $\operatorname{Tr}=3 \times \sigma$, because on the one hand it corresponds to a better definition and separation of filaments in the Serpens Main region (especially in Clusters A and B), and, on the other hand, it makes no appreciable changes on filaments extracted in the Aquila East region.

This algorithm was already used in HGBS papers (Benedettini et al. 2015, 2018). They determine that a source is spatially associated with a filament if its central position falls within the boundary of the filament, as it is evaluated by the algorithm. Following this method, we find that 82 per cent of the entire catalogue sources are on filaments. Of these, 92 per cent are prestellar cores and 8 per cent are unbound cores.

This analysis of the core-filament relationship can be extended by adopting a further definition of spatial association, inspired by the approach of Sewiło et al. (2019) for associating YSOs to filaments, i.e. computing the minimum distance from each source to the nearest filament. From the histogram of these distances (Fig. 16),

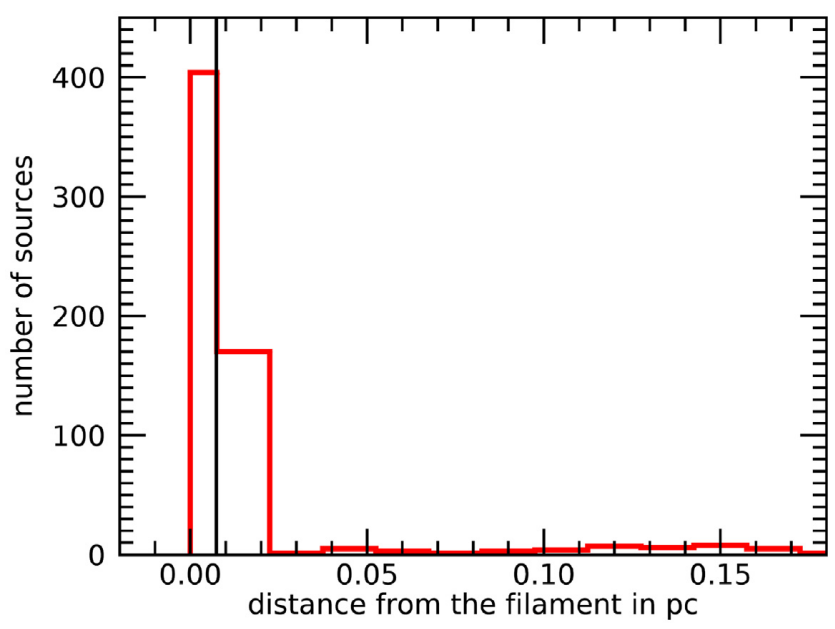

Figure 16. Number of sources at a given distance from the nearest filament. The majority of the on-filament sources appear to be quite close to the spine of the filament. The vertical line corresponds to the resolution element of the column density map.

we note there is a remarkable amount of cores concentrated within $0.03 \mathrm{pc}$ from the spines of filaments, which is the physical distance corresponding to 1 pixel. Therefore, we decide, as an alternative, to associate a core to a filament if it is located at most $0.03 \mathrm{pc}$ from a filament spine.

With the second operational definition, we found that 69 per cent of all detected cores are on-filament within $0.03 \mathrm{pc}$. Note that the number of on-filament sources estimated through the first method ( 82 per cent) is larger than that from the second ( 69 per cent). In the first method, we also labelled as on-filament those sources that are close to the border of the filament, which are ruled out if their distances from the filament spine are considered. We consider the 


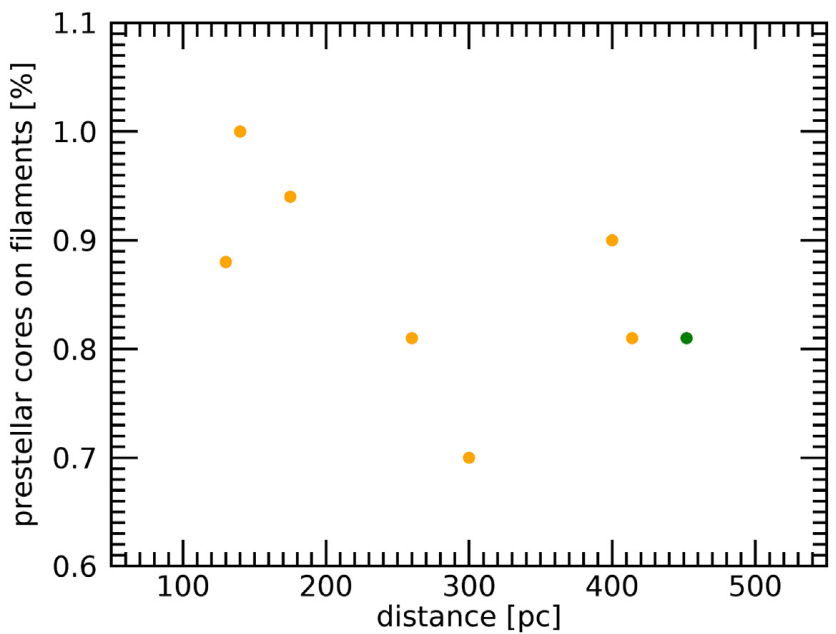

Figure 17. Percentage of on-filament prestellar cores for the HGBS starforming regions reported in Table 6. We use the average value of $175 \mathrm{pc}$ for Lupus cloud and $452 \mathrm{pc}$ for Serpens. The value corresponding to Serpens is in green.

results of the second method more robust, and use them in the following discussion.

The majority of the cores which lie on to filamentary structures are prestellar cores (467 out of 574, 81 per cent), about 14 per cent (77) are protostellar, and only 5 per cent (30) on-filament sources are unbound. This confirm the scenario in which most cores found to be associated with filaments are the ones that will collapse to form stars; instead, cores located outside the filamentary web are more likely to be gravitationally unbound.

To make a comparison between our result and the HGBS previous ones, in Table 6 the percentage of on-filament prestellar cores is reported for each region catalogued so far. For all the analysed regions, this quantity is larger or equal than 67 per cent, being in agreement with the scenario in which stars preferentially form in the filamentary structure. Anyway, there is a spread in this percentage up to 100 per cent, depending on the region and on the different techniques to detect filaments and associate cores to them. This spread, instead, does not depend on the distance, since no correlation is seen in Fig. 17, nor on the type of cores. Therefore, the percentage of on-filament sources is likely to be a combination of a peculiar feature of each specific region and of the filament detection technique adopted, which is different in different works. For this reason, such analysis should be considered only from the qualitative point of view.

In this respect, the Serpens/Aquila East region is configured as one with more on-filament prestellar cores. Fig. 17 also shows that a relevant fraction of the prestellar population is found to lie on filaments. This supports the idea that filamentary structures are preferential places for star formation.

\section{CONCLUSIONS}

In this article, one of the HGBS 'first-generation papers', we presented the Herschel photometric survey (at 70, 160, 250, 350, and $500 \mu \mathrm{m})$ of the Serpens/Aquila East star-forming region, in which two sub-regions can be easily identified: Serpens Main, already studied in the past in different spectral ranges, and Aquila East, so far poorly studied and characterized.

Column density and temperature maps were produced, and their analysis revealed the following: (i) The temperature map contains values between $\sim 14$ and $19 \mathrm{~K}$. The temperature distributions of the two sub-regions appear quite different, however, with Aquila East being on average warmer than the Serpens Main, which is probably more shielded from the interstellar radiation field coming from the Galactic plane.

(ii) The total mass of the region has been computed from the column density map, amounting to about $49400 \mathrm{M}_{\odot}$, larger with respect to estimates in previous works.

From the Herschel maps, we also extracted the first complete catalogue of prestellar sources. Over the observed field, 833 dense cores are detected, of which 709 are starless and 124 are protostellar. We analysed the physical properties of this sub-sample (mass, temperature, and size) and their position with respect the filamentary structure of the cloud. The main results of this analysis are as follows:

(i) From the gravitational stability analysis, 105 cores are found to be gravitationally unbound, and 604 are bound (prestellar).

(ii) The $M$ versus $r$ diagram confirms that Serpens/Aquila East is a low-mass star-forming region. Only 3 prestellar cores might be characterized as compatible with high-mass star formation, but this classification strongly depends on the adopted core-to-star efficiency.

(iii) We find that 45 per cent of the prestellar cores lie in regions populated by Spitzer YSOs, indicating the presence of a mass reservoir for further star formation.

(iv) A negative correlation is found between cores temperature and mean column density, but the coefficients are not consistent with previous estimates for the other HGBS regions.

(v) The prestellar CMF of the Serpens overall region is well fitted by a lognormal function at masses lower than $\sim 2 \mathrm{M}_{\odot}$, and, at higher masses, by a power law whose slope $\gamma=-2.05 \pm 0.34$.

(vi) Both the CMFs restricted to the Serpens Main and Aquila East sub-regions are well fitted in turn by a lognormal function at low masses, and by a power law at high-masses, respectively, with a slope similar to that of the overall region: $-1.95 \pm 0.58$, and $-1.95 \pm 0.41$, respectively.

(vii) We performed the first comparison between CMFs of the HGBS star-forming regions. We notice that the CMF shape strictly depends on the region and the type of cores contained in it. The CMF of unbound cores (Corona Australis and Taurus) shows a shape similar to that of CO clumps. On the contrary, all the regions with a reasonable number of prestellar cores (Aquila Main, Perseus, Orion A and B, Serpens, Cepheus) show a lognormal form in the low-mass regime and the power-law slope at high masses in agreement with the IMF one. This compatibility between prestellar CMF and IMF slopes suggests that the latter is determined by the former, which in turn depends on the cloud fragmentation process.

(viii) We extract the filamentary structure with the tool described in Schisano et al. (2020), finding that 69 per cent of the Serpens cores lie on-filaments. More specifically, 81 per cent of them are prestellar cores, 13 per cent are protostellar cores, and 5 per cent are unbound cores. This strong correspondence between prestellar core locations and filamentary structures supports the idea that filaments are the structures of the molecular clouds in which stars preferentially form.

\section{ACKNOWLEDGEMENTS}

SPIRE has been developed by a consortium of institutes led by Cardiff Univ. (UK) and including: Univ. Lethbridge (Canada); NAOC (China); CEA, LAM (France); IFSI, Univ. Padua (Italy); IAC (Spain); Stockholm Observatory (Sweden); Imperial College London, RAL, UCL-MSSL, UKATC, Univ. Sussex (UK); and Caltech, JPL, NHSC, Univ. Colorado (USA). This development 
has been supported by national funding agencies: CSA (Canada); NAOC (China); CEA, CNES, CNRS (France); ASI (Italy); MCINN (Spain); SNSB (Sweden); STFC, UKSA (UK); and NASA (USA). PACS has been developed by a consortium of institutes led by MPE (Germany) and including UVIE (Austria); KUL, CSL, IMEC (Belgium); CEA, OAMP (France); MPIA (Germany); IFSI, OAP/AOT, OAA/CAISMI, LENS, SISSA (Italy); IAC (Spain). This development has been supported by the funding agencies BMVIT (Austria), ESA-PRODEX (Belgium), CEA/CNES (France), DLR (Germany), ASI (Italy), and CICT/MCT (Spain).

DE, ES, MB, and SM acknowledge financial support by the Agenzia Spaziale Italiana (ASI) under the research contract 201831-HH.0.

DA acknowledges support by FCT/MCTES through national funds (PIDDAC) by the grants UID/FIS/04434/2019 and UIDB/04434/2020.

The computational needs of this project were supported by the GENESIS cluster, which was funded by the project PRIN-INAF 2016 The Cradle of Life - GENESIS-SKA (General Conditions in Early Planetary Systems for the rise of life with SKA).

This work has received support from the European Research Council under the European Union's Seventh Framework Program (ERC Advanced Grant Agreement no. 291294 - 'ORISTARS') and from the French national programmes of CNRS/INSU on stellar and ISM physics (PNPS and PCMI). AB acknowledges the support of the European Union's Horizon 2020 research and innovation program under the Marie Sklodowska-Curie Grant agreement no. 843008 (MUSICA).

\section{DATA AVAILABILITY}

All data are incorporated into the article and its online supplementary material.

\section{REFERENCES}

Alton P. B., Xilouris E. M., Misiriotis A., Dasyra K. M., Dumke M., 2004, A\&A, 425, 109

Alves J., Lombardi M., Lada C. J., 2007, A\&A, 462, L17

André P. et al., 2010, A\&A, 518, L102

André P., Di Francesco J., Ward-Thompson D., Inutsuka S. I., Pudritz R. E., Pineda J. E., 2014, in Beuther H., Klessen R. S., Dullemond C. P., Henning T., eds, Protostars and Planets VI, University of Arizona Press, Tucson. p. 27, preprint (arXiv:1312.6232)

.Benedettini M. et al., 2015, MNRAS, 453, 2036

Benedettini M. et al., 2018, A\&A, 619, A52

Bergin E. A., Tafalla M., 2007, ARA\&A, 45, 339

Bernard J.-P. et al., 2010, A\&A, 518, L88

Bohlin R. C., Savage B. D., Drake J. F., 1978, ApJ, 224, 132

Bonnor W. B., 1956, MNRAS, 116, 351

Bresnahan D. et al., 2018, A\&A, 615, A125

Casali M. M., Eiroa C., Duncan W. D., 1993, A\&A, 275, 195

Chabrier G., 2005, in Corbelli E., Palla F., Zinnecker H., eds, Astrophysics and Space Science Library, Vol. 327, The Initial Mass Function 50 Years Later, Springer, Dordrecht. p. 41 , preprint (astro-ph/0409465)

Chavarria-K. C., de Lara E., Finkenzeller U., Mendoza E. E., Ocegueda J., 1988, A\&A, 197, 151

Di Francesco J., Evans N. J. I., Caselli P., Myers P. C., Shirley Y., Aikawa Y., Tafalla M., 2007, in Reipurth B., Jewitt D., Keil K., eds, Protostars and Planets V, University of Arizona Press, Tucson. p. 17 , preprint (astro-ph/0602379)

Di Francesco J. et al., 2020, ApJ, preprint (arXiv:2010.09894)

Djupvik A. A., André P., Bontemps S., Motte F., Olofsson G., Gålfalk M., Florén H.-G., 2006, A\&A, 458, 789
Dobashi K., 2011, PASJ, 63, S1

Dunham M. M., Crapsi A., Evans N. J., Bourke T. L., Huard T. L., Myers P. C., Kauffmann J., 2008, ApJS, 179, 249

Dunham M. M. et al., 2015, ApJS, 220, 11

Dzib S., Loinard L., Mioduszewski A. J., Boden A. F., Rodríguez L. F., Torres R. M., 2010, ApJ, 718, 610

Dzib S., Loinard L., Mioduszewski A. J., Boden A. F., Rodríguez L. F., Torres R. M., 2011, Rev. Mex. Astron. Astrofís. Conf. Ser., 40, 231

Eiroa C., Djupvik A. A., Casali M. M., 2008, in Reipurth B., ed., The Serpens Molecular Cloud, Astron. Soc. Pac., San Francisco. p. 693

Elia D., Pezzuto S., 2016, MNRAS, 461, 1328

Elia D. et al., 2013, ApJ, 772, 45

Elia D. et al., 2017, MNRAS, 471, 100

Enoch M. L., Glenn J., Evans II N. J., Sargent A. I., Young K. E., Huard T. L., 2007, ApJ, 666, 982

Enoch M. L., Evans II N. J., Sargent A. I., Glenn J., Rosolowsky E., Myers P., 2008, ApJ, 684, 1240

Evans, Neal J. I., Rawlings J. M. C., Shirley Y. L., Mundy L. G., 2001, ApJ, 557,193

Federrath C., 2013, MNRAS, 436, 1245

Griffin M. J. et al., 2010, A\&A, 518, L3

Harvey P. M. et al., 2006, ApJ, 644, 307

Harvey P., Merín B., Huard T. L., Rebull L. M., Chapman N., Evans N. J. I., Myers P. C., 2007, ApJ, 663, 1149

Herczeg G. J. et al., 2019, ApJ, 878, 111

Herschel Science Ground Segment Consortium, 2011, Astrophysics Source Code Library, record ascl:1111.001

Hildebrand R. H., 1983, QJRAS, 24, 267

Kaas A. A. et al., 2004, A\&A, 421, 623

Kainulainen J., Beuther H., Banerjee R., Federrath C., Henning T., 2011, A\&A, 530, A64

Kauffmann J., Pillai T., 2010, ApJ, 723, L7

Kauffmann J., Bertoldi F., Bourke T. L., Evans II N. J., Lee C. W., 2008, A\&A, 487, 993

Kirk J. M. et al., 2013, MNRAS, 432, 1424

Könyves V. et al., 2015, A\&A, 584, A91

Könyves V. et al., 2020, A\&A, 635, A34

Kramer C., Stutzki J., Rohrig R., Corneliussen U., 1998, A\&A, 329, 249

Kroupa P., 2001, MNRAS, 322, 231

Krumholz M. R., McKee C. F., 2008, Nature, 451, 1082

Ladjelate B. et al., 2016, in Jablonka P., André P., van der Tak F., eds, IAU Symposium, Vol. 315, From Interstellar Clouds to Star-Forming Galaxies: Universal Processes? Cambridge University Press, Cambridge,p. E46

Ladjelate B. et al., 2020, A\&A, 638, A74

Lee K. I. et al., 2014, ApJ, 797, 76

Levshakov S. A., Henkel C., Reimers D., Wang M., Mao R., Wang H., Xu Y., 2013, A\&A, 553, A58

Marsh K. A. et al., 2016, MNRAS, 459, 342

McMullin J. P., Mundy L. G., Blake G. A., Wilking B. A., Mangum J. G., Latter W. B., 2000, ApJ, 536, 845

Men'shchikov A., André P., Didelon P., Motte F., Hennemann M., Schneider N., 2012, A\&A, 542, A81

Motte F., Andre P., Neri R., 1998, A\&A, 336, 150

Nakamura F., Dobashi K., Shimoikura T., Tanaka T., Onishi T., 2017, ApJ, 837,154

Offner S. S. R., Clark P. C., Hennebelle P., Bastian N., Bate M. R., Hopkins P. F., Moraux E., Whitworth A. P., 2014, Protostars and Planets VI, University of Arizona Press, Tucson. p. 53

Olmi L., Testi L., 2002, A\&A, 392, 1053

Olmi L. et al., 2013, A\&A, 551, A111

Ortiz-León G. N. et al., 2017, ApJ, 834, 143

Ortiz-León G. N. et al., 2018, ApJ, 869, L33

Ossenkopf V., Henning T., 1994, A\&A, 291, 943

Padoan P., Nordlund Å., 2002, ApJ, 576, 870

Palmeirim P. et al., 2013, A\&A, 550, A38

Pezzuto S. et al., 2020, preprint (arXiv:2010.00006)

Piazzo L., Calzoletti L., Faustini F., Pestalozzi M., Pezzuto S., Elia D., di Giorgio A., Molinari S., 2015, MNRAS, 447, 1471 
Pilbratt G. L. et al., 2010, A\&A, 518, L1

Planck Collaboration et al., 2014, A\&A, 571, A11

Poglitsch A. et al., 2010, A\&A, 518, L2

Polychroni D. et al., 2013, ApJ, 777, L33

Prato L., Rice E. L., Dame T. M., 2008, Where are all the Young Stars in Aquila? Astron. Soc. Pac., San Francisco, p. 18

Roccatagliata V. et al., 2015, A\&A, 584, A119

Roy A. et al., 2014, A\&A, 562, A138

Salpeter E. E., 1955, ApJ, 121, 161

Schisano E. et al., 2014, ApJ, 791, 27

Schisano E. et al., 2020, MNRAS, 492, 5420

Schneider N. et al., 2013, ApJ, 766, L17

Seale J. P., Looney L. W., Wong T., Ott J., Klein U., Pineda J. L., 2012, ApJ, 751,42

Sewiło M. et al., 2019, ApJS, 240, 26

Stamatellos D., Whitworth A. P., Ward-Thompson D., 2007, MNRAS, 379, 1390

Strom S. E., Grasdalen G. L., Strom K. M., 1974, ApJ, 191, 111

Testi L., Sargent A. I., 1998, ApJ, 508, L91

Testi L., Sargent A. I., Olmi L., Onello J. S., 2000, ApJ, 540, L53

White G. J., Casali M. M., Eiroa C., 1995, A\&A, 298, 594

Winston E. et al., 2007, ApJ, 669, 493

Zhang C. Y., Laureijs R. J., Clark F. O., Wesselius P. R., 1988, A\&A, 199, 170

Zucker C., Speagle J. S., Schlafly E. F., Green G. M., Finkbeiner D. P., Goodman A. A., Alves J., 2019, ApJ, 879, 125

\section{SUPPORTING INFORMATION}

Supplementary data are available at $M N R A S$ online.

Table A1. Catalogue of dense cores identified in HGBS maps of Serpens (template, full catalogue only provided online).

Table A2. Derived properties of dense cores identified in HGBS maps of Serpens.

Please note: Oxford University Press is not responsible for the content or functionality of any supporting materials supplied by the authors. Any queries (other than missing material) should be directed to the corresponding author for the article.

\section{APPENDIX A: A CATALOGUE OF DENSE CORES IDENTIFIED WITH HERSCHEL IN THE SERPENS REGION}

Based on our Herschel SPIRE/PACS parallel-mode imaging survey of the Serpens Main and Aquila East star-forming region, we identified a total of 833 dense cores, see Table 4. The catalogue of observed properties of all of these Herschel cores is available in online Table A1. A template of this online catalog is provided below to illustrate its form and content. The derived properties (physical radius, mass, SED dust temperature, peak column density at the resolution of the $500 \mu \mathrm{m}$ data, average column density, peak volume density, and average density) are given in online Table A2 for each core. A portion of this online table is also provided below. The derived properties of the Herscheldetected protostars and YSOs will be published in a forthcoming paper. 


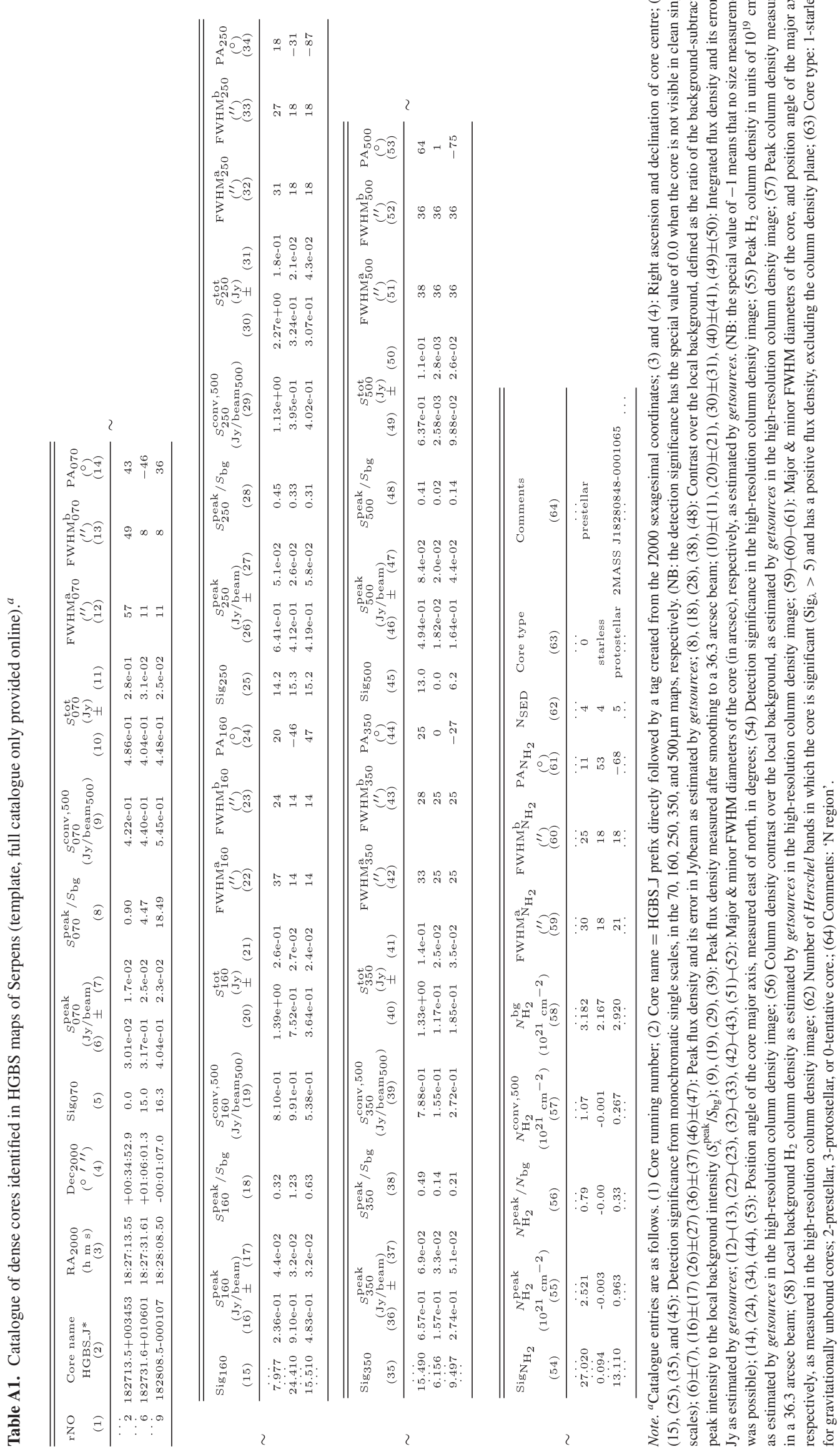




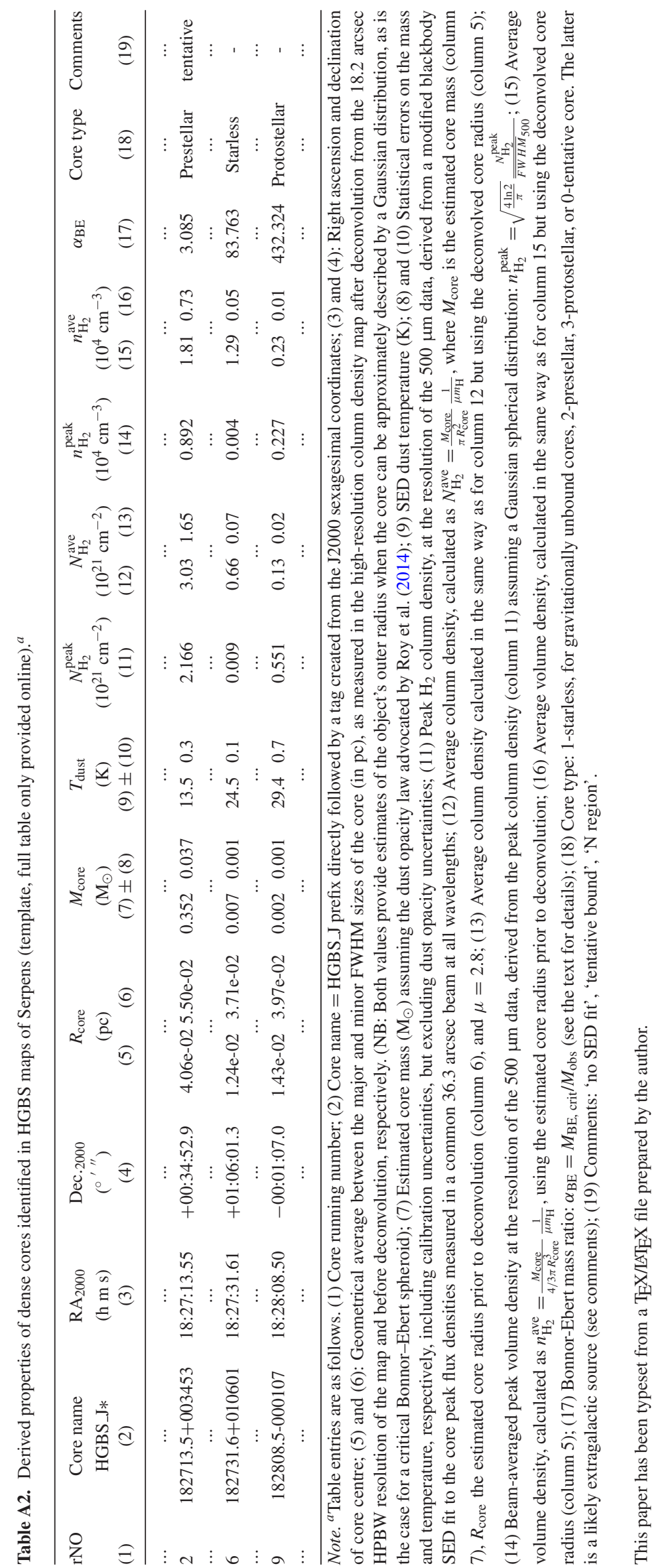

